\title{
The Maxwell-Boltzmann-Euler System with a Massive Scalar Field in All Bianchi Spacetimes
}

\author{
Raoul Domingo Ayissi, Norbert Noutchegueme, and Hugues Paulin Mbeutcha Tchagna \\ Department of Mathematics, Faculty of Science, University of Yaoundé I, P.O. Box 812, Yaoundé, Cameroon \\ Correspondence should be addressed to Raoul Domingo Ayissi; raoulayissi@yahoo.fr
}

Received 6 April 2013; Accepted 17 June 2013

Academic Editor: B. G. Konopelchenko

Copyright (C) 2013 Raoul Domingo Ayissi et al. This is an open access article distributed under the Creative Commons Attribution License, which permits unrestricted use, distribution, and reproduction in any medium, provided the original work is properly cited.

We prove the existence and uniqueness of regular solution to the coupled Maxwell-Boltzmann-Euler system, which governs the collisional evolution of a kind of fast moving, massive, and charged particles, globally in time, in a Bianchi of types I to VIII spacetimes. We clearly define function spaces, and we establish all the essential energy inequalities leading to the global existence theorem.

\section{Introduction}

In this paper, we study the coupled Maxwell-BoltzmannEuler system which governs the collisional evolution of a kind of fast moving, massive, and charged particles and which is one of the basic systems of the kinetic theory.

The spacetimes considered here are the Bianchi of types I to VIII spacetimes where homogeneous phenomena such as the one we consider here are relevant. Note that the whole universe is modeled and particles in the kinetic theory may be particles of ionized gas as nebular galaxies or even cluster of galaxies, burning reactors, and solar wind, for which only the evolution in time is really significant, showing thereafter the importance of homogeneous phenomena.

The relativistic Boltzmann equation rules the dynamics of a kind of particles subject to mutual collisions, by determining their distribution function, which is a nonnegative real-valued function of both the position and the momentum of the particles. Physically, this function is interpreted as the probability of the presence density of the particles in a given volume, during their collisional evolution. We consider the case of instantaneous, localized, binary, and elastic collisions. Here the distribution function is determined by the Boltzmann equation through a nonlinear operator called the collision operator. The operator acts only on the momentum of the particles and describes, at any time, at each point where two particles collide with each other, the effects of the behaviour imposed by the collision on the distribution function, also taking in account the fact that the momentum of each particle is not the same, before and after the collision, with only the sum of their two momenta being preserved.

The Maxwell equations are the basic equations of electromagnetism and determine the electromagnetic field $F$ created by the fast moving charged particles. We consider the case where the electromagnetic field $F$ is generated, through the Maxwell equations by the Maxwell current defined by the distribution function $f$ of the colliding particles, a charge density $e$, and a future pointing unit vector $u$, tangent at any point to the temporal axis.

The matter and energy content of the spacetime is represented by the energy-momentum tensor which is a function of the distribution function $f$, the electromagnetic field $F$, and a massive scalar field $\Phi$, which depends only on the time $t$.

The Euler equations simply express the conservation of the energy-momentum tensor.

The system is coupled in the sense that $f$, which is subject to the Boltzmann equation, generates the Maxwell current in the Maxwell equations and is also present in the Euler equations, whereas the electromagnetic field $F$, which is subject to the Maxwell equations, is in the Lie derivative of $f$ with respect to the vectors field tangent to the trajectories of the particles. $F$ also figures in the Euler equations. 
We consider for the study all the Bianchi of types I to VIII spacetimes, excluding thereby the Bianchi type IX spacetime also called the Kantowski-Sachs spacetime which has the flaw to develop singularities in peculiar finite time and is not convenient for the investigation of global existence of solutions.

The main objective of the present work is to extend the result obtained in [1-3] where the particular case of the Bianchi type I spacetime is investigated. The choice of function spaces and the process of establishing the energy inequalities are highly improved.

The paper is organized as follows.

In Section 2, we introduce the spacetime and we give the unknowns.

In Section 3, we describe the Maxwell-Boltzmann-Euler system.

In Section 4, we define the function spaces and we establish the energy inequalities.

In Section 5, we study the local existence of the solution.

In Section 6, we prove the global existence of the solution.

\section{The Spacetime and the Unknowns}

Greek indexes $\alpha, \beta, \gamma, \ldots$ range from 0 to 3 , and Latin indexes $i, j, k, \ldots$ from 1 to 3 . We adopt the Einstein summation convention:

$$
A^{\alpha} B_{\alpha}=\sum_{\alpha} A^{\alpha} B_{\alpha}
$$

We consider the collisional evolution of a kind of fast moving, massive, and charged particles in the time-oriented Bianchi types 1 to 8 spacetimes $\left(\mathbb{R}^{4}, \widetilde{g}\right)$ and denote by $x^{\alpha}=$ $\left(x^{0}, x^{i}\right)=\left(t, x^{i}\right)$ the usual coordinates in $\mathbb{R}^{4}$, where $x^{0}=$ $t$ represents the time and $\left(x^{i}\right)$ the space; $\tilde{g}$ stands for the given metric tensor of Lorentzian signature $(-,+,+,+)$ which writes

$$
\tilde{g}=-(d t)^{2}+g_{i j} d x^{i} d x^{j},
$$

where $g_{i j}$ are continuously differentiable functions on $\mathbb{R}$, components of a 3-symmetric metric tensor $g=\left(g_{i j}\right)$, whose variable is denoted by $t$.

The expression of the Levi-Civita connection $\nabla$ associated with $\widetilde{g}$, which is

$$
\Gamma_{\alpha \beta}^{\lambda}=\frac{1}{2} \widetilde{g}^{\lambda \mu}\left[\partial_{\alpha} \widetilde{g}_{\mu \beta}+\partial_{\beta} \widetilde{g}_{\alpha \mu}-\partial_{\mu} \widetilde{g}_{\alpha \beta}\right],
$$

gives directly

$$
\begin{gathered}
\Gamma_{i j}^{0}=\frac{1}{2} \partial_{0} g_{i j}, \quad \Gamma_{0 j}^{i}=\frac{1}{2} g^{i l} \partial_{0} g_{j l}, \quad i, j \in\{1,2,3\} \\
\Gamma_{\alpha \beta}^{\lambda}=0 \quad \text { otherwise. }
\end{gathered}
$$

Recall that $\Gamma_{\alpha \beta}^{\lambda}=\Gamma_{\beta \alpha}^{\lambda}$.

We require the assumption that $\partial_{0} g_{i j} / g_{i j}$ are bounded. This implies that there exists a constant $C>0$ such that

$$
\left|\frac{\partial_{0} g_{i j}}{g_{i j}}\right| \leq C \text {. }
$$

As a direct consequence, we have, for $t \in \mathbb{R}^{+}$,

$$
\left|g_{i j}(t)\right| \leq g_{i j}^{0} e^{C t}, \quad\left|\frac{1}{g_{i j}(t)}\right| \leq \frac{1}{g_{i j}^{0}} e^{C t},
$$

where $g_{i j}^{0}=g_{i j}(0)$.

The massive particles have a rest mass $m>0$, normalized to the unity, that is, $m=1$. We denote by $T\left(\mathbb{R}^{4}\right)$ the tangent bundle of $\mathbb{R}^{4}$ with coordinates $\left(x^{\alpha}, p^{\beta}\right)$, where $p=$ $\left(p^{\beta}\right)=\left(p^{0}, \bar{p}\right)$ stands for the momentum of each particle and $\bar{p}=\left(p^{i}\right), i=1,2,3$. Really the charged particles move on the future sheet of the mass shell or the mass hyperboloid $P\left(\mathbb{R}^{4}\right) \subset T\left(\mathbb{R}^{4}\right)$, whose equation is $P_{x}(p): \tilde{g}_{x}(p, p)-1$ or, equivalently, using expression (2) of $\tilde{g}$ :

$$
p^{0}=\sqrt{1+g_{i j} p^{i} p^{j}}
$$

where the choice $p^{0}>0$ symbolizes the fact that, naturally, the particles eject towards the future.

Setting

$$
\varrho=\sqrt{\sum_{i=1}^{3}\left(p^{i}\right)^{2}}=\varrho(\bar{p}),
$$

if $\varrho>1$, the relations (6) and (7) also show that in any interval $[0, T], T>0$ :

$$
A p^{0} \leq \varrho \leq B p^{0}
$$

where $A=A(T)>0, B=B(T)>0$ are constants.

The invariant volume element in $P_{x}(p)$ reads

$$
\omega_{p}=|\tilde{g}|^{1 / 2} \frac{d p^{1} d p^{2} d p^{3}}{p^{0}},
$$

where

$$
|\tilde{g}|=\left|\operatorname{det} g_{\alpha \beta}\right|
$$

We denote by $f$ the distribution function which measures the probability of the presence of particles in the plasma. $f$ is a nonnegative unknown real-valued function of both the position $\left(x^{\alpha}\right)$ and the 4 -momentum of the particles $p=\left(p^{\alpha}\right)$, so:

$$
\begin{aligned}
& f: T\left(\mathbb{R}^{4}\right) \approx \mathbb{R}^{4} \times \mathbb{R}^{4} \longrightarrow \mathbb{R}^{+}, \\
& \left(x^{\alpha}, p^{\alpha}\right) \longmapsto f\left(x^{\alpha}, p^{\alpha}\right) \in \mathbb{R}^{+} .
\end{aligned}
$$

We define a scalar product on $\mathbb{R}^{3}$ by setting for $p=$ $\left(p^{0}, \bar{p}\right)=\left(p^{0}, p^{i}\right)$ and $q=\left(q^{0}, \bar{q}\right)=\left(q^{0}, q^{i}\right)$ :

$$
\bar{p} \cdot \bar{q}=g_{i j} p^{i} q^{j}
$$

In this paper we consider the homogeneous case for which $f$ depends only on the time $x^{0}=t$ and $\bar{p}$. According to the Laplace law, the fast moving and charged particles 
create an unknown electromagnetic field $F$ which is a 2closed antisymmetric form and locally writes

$$
F=F_{\alpha \beta} d x^{\alpha} \wedge d x^{\beta}
$$

So in the homogeneous case we consider

$$
F_{\alpha \beta}: \mathbb{R} \longrightarrow \mathbb{R}, \quad t \longmapsto F_{\alpha \beta}(t) \in \mathbb{R} .
$$

In the presence of the electromagnetic field $F$, the trajectories $s \mapsto\left(x^{\alpha}(s), p^{\alpha}(s)\right)$ of the charged particles are no longer the geodesics of spacetime $\left(\mathbb{R}^{4}, g\right)$ but the solutions of the differential system:

$$
\frac{d x^{\alpha}}{d s}=p^{\alpha} ; \quad \frac{d p^{\alpha}}{d s}=P^{\alpha},
$$

where

$$
P^{\alpha}=P(F, f)=-\Gamma_{\lambda \mu}^{\alpha} p^{\lambda} p^{\mu}+e p^{\beta} F_{\beta}^{\alpha},
$$

where $e=e(t)$ denotes the charge density of particles.

Notice that the differential system (16) shows that the vectors field $X(F)$ defined locally by

$$
X(F)=\left(p^{\alpha}, P^{\alpha}(F)\right),
$$

where $P^{\alpha}$ is given by (17), is tangent to the trajectories.

The charged particles also create a current $J=\left(J^{\beta}\right), \beta=$ $0,1,2,3$, called the Maxwell current which we take in the form

$$
J^{\beta}=\int_{\mathbb{R}^{3}} p^{\beta} f \omega_{p}-e u^{\beta}
$$

in which $u=\left(u^{\beta}\right)$ is a unit future pointing timelike vector, tangent to the time axis at any point, which means that $u^{0}=1$, $u^{i}=u_{i}=0$, and $i=1,2,3$. The particles are then supposed to be spatially at rest.

The electromagnetic field $F=\left(F^{0 i}, F_{i j}\right)$, where $F^{0 i}$ and $F_{i j}$ stand for the electric and magnetic parts, respectively, is subject to the Maxwell equations.

\section{The Maxwell-Boltzmann-Euler System in $F, f$, and $\Phi$}

3.1. The Maxwell Equations in F. The Maxwell system in F can be written, using the covariant notation:

$$
\begin{gathered}
\nabla_{\alpha} F^{\alpha \beta}=J^{\beta} \\
\nabla_{\alpha} F_{\beta \gamma}+\nabla_{\beta} F_{\gamma \alpha}+\nabla_{\gamma} F_{\alpha \beta}=0 .
\end{gathered}
$$

Equations (20) and (21) are, respectively, the first and second groups of the Maxwell equations, and $\nabla_{\alpha}$ stands for the convariant derivative in $g$. In $(20), J^{\beta}$ represents the Maxwell current we take in the form (19). Now the well-known identity $\nabla_{\alpha} \nabla_{\beta} F^{\alpha \beta}=0$ imposes, given (20), that the current $j^{\beta}$ is always subject to the conservation law:

$$
\nabla_{\beta} J^{\beta}=0
$$

However using $\beta=0$ in (20), we obtain since $F=F(t)$, $F^{\alpha \lambda}=-F^{\lambda \alpha}$ and by (4) that

$$
J^{0}=0 \text {. }
$$

By (23), the expression (19) of $J^{\beta}$ in which we set $\beta=0$ then allows to compute $e$ and gives, since $u^{0}=1$,

$$
e(t)=\int_{\mathbb{R}^{3}} f(t, \bar{p})(\operatorname{det} g)^{1 / 2} d \bar{p},
$$

which shows that $f$ determines $e$.

The second set (21) of the Maxwell equations is identically satisfied since $F=F(t)$, and the first set reduces to $\partial F_{i j}=0$. Then $F_{i j}$ is constant and

$$
F_{i j}=F_{i j}(0)=\varphi_{i j}
$$

This physically shows that the magnetic part of $F$ does not evolve and stays in its primitive state. It remains to determine the electric part $F^{0 i}$.

Writing (19) for $\beta=i$, using (4), $\omega_{p}=$ $|g|^{1 / 2}\left(d p^{1} d p^{2} d p^{3} / p^{0}\right)$, and $u^{i}=0$, implies that

$$
J^{i}=\int_{\mathbb{R}^{3}} \frac{p^{i} f(t, \bar{p})(\operatorname{det} g)^{1 / 2}}{p^{0}} d \bar{p} .
$$

By (20), we obtain the linear o.d.e in $F^{0 i}$ which writes

$$
\partial_{0} F^{0 i}-H F^{0 i}=\int_{\mathbb{R}^{3}} \frac{p^{i} f(t, \bar{p})(\operatorname{det} g)^{1 / 2}}{p^{0}} d \bar{p} .
$$

Remark 1. In (27), the expression

$$
H=-\frac{1}{2} g^{k l} \partial_{0} g_{k l}
$$

represents the second fundamental form in $\left(\mathbb{R}^{3}, g\right)$. Really $H$ is the trace of the 2-symmetric tensor $K=\left(K_{i j}\right)$ where $K_{i j}=$ $-(1 / 2) \partial_{0} g_{i j} . H$ is called the middle curvature of $\left(\mathbb{R}^{4}, \tilde{g}\right)$. Since $g=\left(g_{i j}\right)$ is given, so is $H$.

3.2. The Relativistic Boltzmann Equation in $f$. The relativistic Boltzmann equation in $f$, for charged particles in the Bianchi types 1 to 8 spacetimes, can be written:

$$
L_{X} f=Q(f, f),
$$

where $L_{X}$ is the Lie derivative of $f$ with respect to the vectors field $X(F)$ defined by $(18)$ and $Q(f, f)$, the collision operator we now introduce.

According to Lichnerowicz and Chernikov, we consider a scheme, in which, at a given position $\left(t, x^{i}\right)$, only two particles collide with each other, without destroying each other, with the collision affecting only the momentum of each particle, which changes after shock, only the sum of the two momenta being preserved. If $p, q$ stand for the two momenta before the shock and $p^{\prime}, q^{\prime}$ for the two momenta after the shock, then we have

$$
p+q=p^{\prime}+q^{\prime} .
$$


The collision operator $Q$ is then defined, using functions $f$ and $g$ on $\mathbb{R}^{3}$, and the previous notations by

$$
Q(f, g)=Q^{+}(f, g)-Q^{-}(f, g),
$$

where

$$
\begin{gathered}
Q^{+}(f, g)=\int_{\mathbb{R}^{3}} \omega_{\bar{q}} \int_{S^{2}} f\left(\bar{p}^{\prime}\right) g\left(\bar{q}^{\prime}\right) \sigma\left(t, \bar{p}, \bar{q}, \bar{p}^{\prime}, \bar{q}^{\prime}, \Omega\right) d \Omega \\
Q^{-}(f, g)=\int_{\mathbb{R}^{3}} \omega_{\bar{q}} \int_{S^{2}} f(\bar{p}) g(\bar{q}) \sigma\left(t, \bar{p}, \bar{q}, \bar{p}^{\prime}, \bar{q}^{\prime}, \Omega\right) d \Omega
\end{gathered}
$$

whose elements we now introduce step by step, specifying properties and hypotheses we adopt:

(i) $S^{2}$ is the unit sphere of $\mathbb{R}^{3}$, whose area element is denoted by $d \Omega$;

(ii) $\sigma$ is a nonnegative continuous real-valued function of all its arguments, called the collision kernel or the cross-section of the collisions, on which we require the boundedness and Lipschitz continuity assumptions, in which $C_{1}>0$ is a constant:

$$
\begin{gathered}
0 \leq \sigma(t, \bar{p}, \bar{q}, \Omega) \leq C_{1} \\
\left|\sigma\left(t, \bar{p}_{1}, \bar{q}, \bar{p}^{\prime}, \bar{q}^{\prime}, \Omega\right)-\sigma\left(t, \bar{p}_{2}, \bar{q}, \bar{p}^{\prime}, \bar{q}^{\prime}, \Omega\right)\right| \\
\leq C_{1}\left\|\bar{p}_{1}-\bar{p}_{2}\right\|,
\end{gathered}
$$

where $\|\bar{p}\|=\left(\sum_{i=1}^{3}\left(p^{i}\right)^{2}\right)^{1 / 2}=\varrho$ is the norm in $\mathbb{R}^{3}$.

(iii) The conservation law $p+q=p^{\prime}+q^{\prime}$ splits into

$$
\begin{gathered}
p^{0}+q^{0}=p^{\prime 0}+q^{\prime 0}, \\
\bar{p}+\bar{q}=\bar{p}^{\prime}+\bar{q}^{\prime} .
\end{gathered}
$$

Equation (34) expresses, using (7), the conservation of the quantity:

$$
\widetilde{e}=\sqrt{1+g_{i j} p^{i} p^{j}}+\sqrt{1+g_{i j} q^{i} q^{j}}
$$

called the elementary energy of the unit rest mass particles; we can interpret (35) by setting, following Glassey and Strauss in [4, equation (42)],

$$
\begin{aligned}
& \bar{p}^{\prime}=\bar{p}+a(\bar{p}, \bar{q}, \Omega) \Omega \\
& \bar{q}^{\prime}=\bar{q}-a(\bar{p}, \bar{q}, \Omega) \Omega
\end{aligned} \quad\left(\Omega \in S^{2}\right)
$$

in which $a(\bar{p}, \bar{q}, \Omega)$ is a real-valued function. Using (7) to express $p^{\prime 0}, q^{\prime 0}$ in terms of $\bar{p}^{\prime}, \bar{q}^{\prime}$ and next (37) to express $\bar{p}^{\prime}$, $\bar{q}^{\prime}$ in terms of $\bar{p}, \bar{q}$, we prove that (34) leads to a quadratic equation in $a$, which solves to give the only nontrivial solution:

$$
a(\bar{p}, \bar{q}, \Omega)=\frac{2 p^{0} q^{0} \widetilde{e} \Omega \cdot(\hat{\bar{q}}-\hat{\bar{p}})}{(\widetilde{e})^{2}-[\Omega \cdot(\bar{p}+\bar{q})]^{2}}
$$

in which $\widehat{\bar{p}}=\bar{p} / p^{0}, \widetilde{e}$ is given by (36), and the $\operatorname{dot}(\cdot)$ is the scalar product defined by (13).

It consequently appears, using (37), that the functions in the integrals (32) depend only on $\bar{p}, \bar{q}, \Omega$ and that these integrals with respect to $\bar{q}$ and $\Omega$ give functions $Q^{+}(f, g)$ and $Q^{-}(f, g)$ of the single variable $\bar{p}$.

Using now the usual properties of the determinants, we compute the Jacobian of the change of variables $(\bar{p}, \bar{q}) \mapsto$ $\left(\bar{p}^{\prime}, \bar{q}^{\prime}\right)$ defined by (37) and find

$$
\frac{\partial\left(p^{\prime}, q^{\prime}\right)}{\partial(p, q)}=-\frac{p^{\prime 0} q^{\prime 0}}{p^{0} q^{0}}
$$

But $f=f(t, \bar{p})$, so using (7), the Boltzmann equation (29) leads to the following form:

$$
\frac{\partial f}{\partial t}+\frac{P^{i}}{p^{0}} \frac{\partial f}{\partial p^{i}}=\frac{1}{p^{0}} Q(f, f) .
$$

3.3. The Euler Equations. The Euler equations only express the conservation of the energy-momentum tensor $T_{\alpha \beta}$ and write

$$
\nabla_{\alpha} T^{\alpha \beta}=0
$$

In (41),

$$
T_{\alpha \beta}=T_{\alpha \beta}^{1}+\tau_{\alpha \beta}+T_{\alpha \beta}^{2}
$$

where

$$
\begin{gathered}
T_{\alpha \beta}^{1}=\int_{\mathbb{R}^{3}} \frac{p_{\alpha} p_{\beta} f(t, \bar{p})(\operatorname{det} g)^{1 / 2} d \bar{p}}{p^{0}}, \\
\tau_{\alpha \beta}=-\frac{\tilde{g}_{\alpha \beta}}{4} F^{\lambda \mu} F_{\lambda \mu}+F_{\beta \lambda} F_{\alpha}^{\lambda}, \\
T_{\alpha \beta}^{2}=\nabla_{\alpha} \Phi \nabla_{\beta} \Phi-\frac{\tilde{g}_{\alpha \beta}}{2}\left[\nabla^{\lambda} \Phi \nabla_{\lambda} \Phi+m_{0}^{2} \Phi^{2}\right] .
\end{gathered}
$$

(i) $T_{\alpha \beta}^{1}$ is the energy-momentum tensor associated with $f$;

(ii) $\tau_{\alpha \beta}$ is the Maxwell tensor associated with $F$;

(iii) $T_{\alpha \beta}^{2}$ is the energy-momentum tensor associated with the scalar field $\Phi$ whose mass is denoted by $m_{0}$, with $m_{0}>0$.

Equation (42) shows that (41) writes

$$
\nabla_{\alpha} T^{1, \alpha \beta}+\nabla_{\alpha} \tau^{\alpha \beta}+\nabla_{\alpha} T^{2, \alpha \beta}=0
$$

But it is proved in [5] that if $f$ verifies the Boltzmann equation (40), then $T^{1, \alpha \beta}$ defined by (43) verifies $\nabla_{\alpha} T^{1, \alpha \beta}=0$; (46) reduces then to

$$
\nabla_{\alpha} \tau^{\alpha \beta}+\nabla_{\alpha} T^{2, \alpha \beta}=0 .
$$


Now, using (21), we have

$$
\nabla_{\alpha} \tau^{\alpha \beta}=F_{\lambda}^{\beta} \nabla_{\alpha} F^{\alpha \lambda}
$$

and using (45),

$$
\nabla_{\alpha} T^{2, \alpha \beta}=\nabla^{\beta} \Phi\left(\square_{\tilde{g}} \Phi-m_{0}^{2}\right)
$$

where $\square_{\widetilde{g}}=\nabla_{\alpha} \nabla^{\alpha}$ is the D'Alembertian.

We deduce from (20), (48), and (49) that the Euler equations (41) are satisfied if $\Phi$ verifies the second-order differential equation:

$$
\nabla^{\beta} \Phi\left(\square_{\tilde{g}} \Phi-m_{0}^{2}\right)+F_{\lambda}^{\beta} \nabla_{\alpha} F^{\alpha \lambda}=0
$$

For $\beta=i$, (50) leads to the constraints system:

$$
F_{j}^{i} \int_{\mathbb{R}^{3}} \frac{p^{j}}{p^{0}} f(t, \bar{p})(\operatorname{det} g)^{1 / 2} d \bar{p}=0, \quad i=1,2,3,
$$

between the unknown functions $F$ and $f$, constraints which we have to solve in what is to follow.

For $\beta=0$, (50) leads to a nonlinear differential equation of second order:

$$
\dot{\Phi}\left(\ddot{\Phi}-H \dot{\Phi}+m_{0}^{2} \Phi\right)=g_{i j} F^{0 j} \int_{\mathbb{R}^{3}} \frac{p^{j}}{p^{0}} f(t, \bar{p})(\operatorname{det} g)^{1 / 2} d \bar{p}
$$

where $H$ is defined in (27).

Setting in (52)

$$
U=\frac{1}{2}(\dot{\Phi})^{2}
$$

it comes that

$$
\begin{gathered}
U \geq 0 \\
\dot{\Phi}= \pm \sqrt{2 U} .
\end{gathered}
$$

One supposes in what follows that $\Phi$ is continuously differentiable, is not a constant, and is decreasing. This implies that

$$
\begin{gathered}
\dot{\Phi}=-\sqrt{2 U} \\
\Phi(t) \leq \Phi(0), \quad t \in \mathbb{R}^{+} .
\end{gathered}
$$

Equation (52) is then equivalent to the nonlinear firstorder differential system given as follows:

$$
\begin{gathered}
\dot{\Phi}=-\sqrt{2 U} \\
\dot{U}=2 H U+m_{0}^{2} \Phi \sqrt{2 U}+g_{i j} E^{i} \int_{\mathbb{R}^{3}} \frac{p^{j}}{p^{0}} f(t, \bar{p})(\operatorname{det} g)^{1 / 2} d \bar{p}
\end{gathered}
$$

where $E^{i}=F^{0 i}$.
3.4. The Coupled System. From (17), using (4), we obtain

$$
\frac{P^{i}}{p^{0}}=-2 \Gamma_{0 j}^{i} p^{j}+e\left[-E^{i}+g^{i j} \frac{p^{k} F_{k j}}{p^{0}}\right], \quad i=1,2,3 .
$$

Using (24), (27), (40), (56), and (57), the MaxwellBoltzmann-Euler system in $(F, f, \Phi, U)$ reduces to the following form:

$$
\begin{gathered}
\dot{E}^{i}=H E^{i}+\int_{\mathbb{R}^{3}} \frac{p^{i} f(t, \bar{p})(\operatorname{det} g)^{1 / 2}}{p^{0}} d \bar{p} \\
\frac{\partial f}{\partial t}+\left[-2 \Gamma_{0 j}^{i} p^{j}+\left(-E^{i}+g^{i j} \frac{p^{k} \varphi_{k j}}{p^{0}}\right)\right. \\
\left.\times \int_{\mathbb{R}^{3}} f(t, \bar{p})(\operatorname{det} g)^{1 / 2} d \bar{p}\right] \frac{\partial f}{\partial p^{i}}=\frac{1}{p^{0}} Q(f, f) \\
\dot{\Phi}=-\sqrt{2 U}, \\
\dot{U}=2 H U+m_{0}^{2} \Phi \sqrt{2 U}+g_{i j} E^{j} \int_{\mathbb{R}^{3}} \frac{p^{i}}{p^{0}} f(t, \bar{p})(\operatorname{det} g)^{1 / 2} d \bar{p}
\end{gathered}
$$

which is an integrodifferential system to solve in what is to follow.

We are searching a solution $(F, f, \Phi, U)$ of the Cauchy problem (59)-(60)-(61)-(62) globally in time on $[0,+\infty$ [ for the initial data:

$$
\begin{aligned}
E^{i}(0)=E_{0}^{i} ; & F_{i j}(0)=\varphi_{i j}, & f(0, \bar{p})=f_{0}(\bar{p}), \\
\bar{p} \in \mathbb{R}^{3} ; & \Phi(0)=\Phi_{0} ; & U(0)=U_{0} .
\end{aligned}
$$

3.5. The Problem of Constraints. We must find a nontrivial solution $(F, f, \Phi, U)$ of the Cauchy problem (59)-(60)-(61)(62) satisfying the system (51) of constraints which writes after computation

$$
\begin{gathered}
\left(g^{12} J^{1}-g^{11} J^{2}\right) \varphi_{21}+\left(g^{13} J^{1}-g^{11} J^{3}\right) \varphi_{31} \\
+\left(g^{13} J^{2}-g^{12} J^{3}\right) \varphi_{32}=0 \\
\left(g^{22} J^{1}-g^{21} J^{2}\right) \varphi_{21}+\left(g^{23} J^{1}-g^{21} J^{3}\right) \varphi_{31} \\
\quad+\left(g^{23} J^{2}-g^{22} J^{3}\right) \varphi_{32}=0 \\
\left(g^{32} J^{1}-g^{31} J^{2}\right) \varphi_{21}+\left(g^{33} J^{1}-g^{31} J^{3}\right) \varphi_{31} \\
+\left(g^{33} J^{2}-g^{32} J^{3}\right) \varphi_{32}=0
\end{gathered}
$$

\section{Function Spaces and Energy Inequalities}

We define now the function spaces in which we are searching the solution to the Maxwell-Boltzmann-Euler system. We also establish some useful energy estimations. 
Definition $2\left(E_{s}^{m}\left(0, T, \mathbb{R}^{3}\right)\right)$. Let $T>0, m \in \mathbb{N}, s \in \mathbb{R}$ be given. We define $E_{s}^{m}\left(\mathbb{R}^{3}\right)$ as

$E_{s}^{m}\left(\mathbb{R}^{3}\right)$

$$
\begin{aligned}
= & \left\{h: \mathbb{R}^{3} \longrightarrow \mathbb{R},(1+\varrho)^{s+|\beta|} \partial_{\bar{p}}^{\beta} h \in L^{2}\left(\mathbb{R}^{3}\right),\right. \\
& |\beta| \leq m\} .
\end{aligned}
$$

$E_{s}^{m}\left(\mathbb{R}^{3}\right)$ will be endowed with the norm

$$
\|h\|_{E_{s}^{m}\left(\mathbb{R}^{3}\right)}=\max _{0 \leq|\beta| \leq m}\left\|(1+\varrho)^{s+|\beta|} \partial_{\bar{p}}^{\beta} h\right\|_{L^{2}\left(\mathbb{R}^{3}\right)} .
$$

$\bar{E}_{s}^{m}\left(\mathbb{R}^{3}\right)$ will be the completion of $E_{s}^{m}\left(\mathbb{R}^{3}\right)$ in the norm $\|\cdot\|_{E_{s}^{m}\left(\mathbb{R}^{3}\right)} \cdot$

We also define

$$
\begin{aligned}
E_{s}^{m}\left(0, T, \mathbb{R}_{\bar{p}}^{3}\right) & \\
= & \left\{y \in \mathscr{C}\left([0, T] ; \mathscr{E}\left(\mathbb{R}_{\bar{p}}^{3}\right)\right),\right. \\
& \left.(1+\varrho)^{s+|\beta|} \partial_{\bar{p}}^{\beta} y(t, \cdot) \in L^{2}\left(\mathbb{R}_{\bar{p}}^{3}\right),|\beta| \leq m, 0 \leq t \leq T\right\} .
\end{aligned}
$$

Endowed with the norm

$$
\|\| y\left\|_{E_{s}^{m}\left(0, T, \mathbb{R}_{\bar{p}}^{3}\right)}=\max _{0 \leq|\beta| \leq m} \sup _{t \in[0, T]}\right\|(1+\varrho)^{s+|\beta|} \partial_{\bar{p}}^{\beta} y(t, \cdot) \|_{L^{2}\left(\mathbb{R}_{\bar{p}}^{3}\right)},
$$

$E_{s}^{m}\left(0, T, \mathbb{R}^{3}\right)$ is a Banach space.

$H_{s}^{m}\left(0, T, \mathbb{R}_{\bar{p}}^{3}\right)$ will be the completion of $E_{s}^{m}\left(0, T, \mathbb{R}_{\bar{p}}^{3}\right)$ for the norm $|\|\cdot\||_{E_{s}^{m}\left(0, T, \mathbb{R}_{\bar{p}}^{3}\right)}$.

For $r>0$ to be given, we define

$$
E_{s, r}^{m}=\left\{y \in E_{s}^{m}\left(0, T, \mathbb{R}^{3}\right), \quad y \geq 0 \text { a.e, }\|\| y \|_{E_{s}^{m}\left(0, T, \mathbb{R}_{\bar{p}}^{3}\right)} \leq r\right\} .
$$

Endowed with the induced distance by the norm $|\|\cdot\||_{E_{s}^{m}\left(0, T, \mathbb{R}_{\bar{p}}^{3}\right)}, \quad E_{s, r}^{m}$ is a complete metric subspace of $E_{s}^{m}\left(0, T, \mathbb{R}_{\bar{p}}^{3}\right)$.

Remark 3. If $m=0$, then $y \in E_{s}^{m}\left(\mathbb{R}^{3}\right) \Leftrightarrow(1+\varrho)^{s} y \in L^{2}\left(\mathbb{R}^{3}\right)$, so $E_{\mathrm{s}}^{0}\left(\mathbb{R}^{3}\right)$ will be denoted by $L_{s}^{2}\left(\mathbb{R}^{3}\right)$.

Remark 4. The reasons for the choice of the function space $H_{s}^{m}\left(0, T, \mathbb{R}_{\bar{p}}^{3}\right)$ for $m=3$ and $d>5 / 2$.

With the objective of the present work being the existence of solution to the Maxwell-Boltzmann-Euler system, and particularly the Boltzmann equation (40), we are searching a function $f=f(t, \bar{p})$ which is continuously differentiable; in particular we can search $f=f(t, \cdot)$ belonging to the space $\mathscr{C}_{b}^{1}\left(\mathbb{R}_{\bar{p}}^{3}\right)$.

We want to use the Faedo-Galerkin method which is applied for separable Hilbert spaces. That is the case for the Sobolev spaces $H_{s}^{m}\left(0, T, \mathbb{R}_{\bar{p}}^{3}\right), m \in \mathbb{N}$.
We need then to find an integer $m$ such that

$$
H^{m}\left(\mathbb{R}_{\bar{p}}^{3}\right) \hookrightarrow \mathscr{C}_{b}^{1}\left(\mathbb{R} \frac{3}{p}\right) .
$$

But we know by the Sobolev theorems that

$$
W_{p}^{m}\left(\mathbb{R}^{n}\right) \hookrightarrow \mathscr{E}^{k}\left(\mathbb{R}^{n}\right), \quad m>k+\frac{n}{p} .
$$

Since in our case we have $n=3, p=2$, and $k=1\left(W_{2}^{m}=\right.$ $\left.H^{m}\right)$, we must choose $m$ such that

$$
m>1+\frac{3}{2}=\frac{5}{2} .
$$

The smallest integer $m$ satisfying $m>5 / 2$ is naturally $m=3$.

Consequently we have

$$
H_{d}^{3}\left(\mathbb{R}_{\bar{p}}^{3}\right) \hookrightarrow H^{3}\left(\mathbb{R}^{3}\right) \hookrightarrow \mathscr{C}_{b}^{1}\left(\mathbb{R}_{\bar{p}}^{3}\right) .
$$

Furthermore if

$$
d>\frac{5}{2}
$$

then

$$
H_{d}^{m}\left(\mathbb{R}_{\bar{p}}^{3}\right) \hookrightarrow L_{d}^{2}\left(\mathbb{R}_{\bar{p}}^{3}\right) \hookrightarrow L_{2}^{1}\left(\mathbb{R}_{\bar{p}}^{3}\right),
$$

where $L_{2}^{1}\left(\mathbb{R}_{\frac{p}{p}}^{3}\right)$ is defined in [1].

It then results that

$$
H_{d}^{m}\left(\mathbb{R}_{\bar{p}}^{3}\right) \cap L_{2}^{1}\left(\mathbb{R}_{\bar{p}}^{3}\right)=H_{d}^{m}\left(\mathbb{R}_{\bar{p}}^{3}\right) .
$$

We can now state the following results which will be fundamental.

Lemma 5. There exists a real number $T>0$ such that

$$
(\widetilde{e})^{2}-[\Omega \cdot(\bar{p}+\bar{q})]^{2}>2 .
$$

Furthermore, one has

$$
\begin{aligned}
& (\widetilde{e})^{2}-[\Omega \cdot(\bar{p}+\bar{q})]^{2} \geq\left(1-\sum_{i, j} g_{i j}\right)\left(p^{0}\right)^{2} \\
& (\widetilde{e})^{2}-[\Omega \cdot(\bar{p}+\bar{q})]^{2} \geq\left(1-\sum_{i, j} g_{i j}\right)\left(q^{0}\right)^{2} \\
& (\widetilde{e})^{2}-[\Omega \cdot(\bar{p}+\bar{q})]^{2} \geq\left(1-\sum_{i, j} g_{i j}\right) p^{0} q^{0},
\end{aligned}
$$

and the function $(\bar{p}, \bar{q}, \Omega) \mapsto D_{\bar{p}}^{\beta} c(\bar{p}, \bar{q}, \Omega), 1 \leq|\beta| \leq 3$ is bounded.

Proof. See [2]. 
Proposition 6. Let $d>5 / 2,\|\sigma\|_{L^{1}\left(\mathbb{R}^{3} \times S^{2}\right)} \in L^{\infty}\left(\mathbb{R}^{3}\right)$ and $\left(\partial^{\beta} \sigma\right)(1+|\bar{p}|)^{|\beta|-1} \in L^{\infty}\left(\mathbb{R}^{3} \times \mathbb{R}^{3} \times S^{2}\right),|\beta| \leq 3$ be given. has

If $f, g \in H_{d}^{3}\left(\mathbb{R}^{3}\right)$, then $\left(1 / p^{0}\right) Q(f, g) \in H_{d}^{3}\left(\mathbb{R}^{3}\right)$, and one

$$
\left\|\frac{1}{p^{0}} Q(f, g)\right\|_{H_{d}^{3}\left(\mathbb{R}^{3}\right)} \leq C\|f\|_{H_{d}^{3}\left(\mathbb{R}^{3}\right)}\|g\|_{H_{d}^{3}\left(\mathbb{R}^{3}\right)},
$$

where $C=C(T)>0$.

Moreover

$$
\begin{aligned}
& \left\|\frac{1}{p^{0}} Q(f, f)-\frac{1}{p^{0}} Q(g, g)\right\|_{H_{d}^{3}\left(\mathbb{R}^{3}\right)} \\
& \quad \leq C\left(\|f\|_{H_{d}^{3}\left(\mathbb{R}^{3}\right)}+\|g\|_{H_{d}^{3}\left(\mathbb{R}^{3}\right)}\right)\|f-g\|_{H_{d}^{3}\left(\mathbb{R}^{3}\right)} .
\end{aligned}
$$

Proof. We simply use Lemma 5. For the details, see [2].

Proposition 7. Letd $>5 / 2, f \in H_{d}^{0}\left(\mathbb{R}^{3}\right)$ be given. Then $\left|J^{i}\right| \leq$ $C(\operatorname{det} g)^{1 / 2}\|f\|_{H_{d}^{0}\left(\mathbb{R}^{3}\right)}, i=1,1,3$.

Proof. See [2].

Remark 8. The hypothesis of Proposition 6 concerning the collision kernel $\sigma$ is a supplementary hypothesis for the investigation of the solution to the Boltzmann equation.

In what is to follow, we are searching the local existence and the uniqueness of the solution to the Cauchy problem (59)-(60)-(61)-(62) in a function space which we will precise, applying the standard theory of first-order differential systems.

The framework we will refer to for $f$ is $H_{d}^{3}\left(0, T, \mathbb{R}_{\bar{p}}^{3}\right)$.

The framework we will refer to for $\bar{E}$ is $\mathbb{R}^{3}$, whose norm is denoted by $\|\cdot\|$ or $\|\cdot\|_{\mathbb{R}^{3}}$ :

$$
\begin{aligned}
& \mathscr{C}\left(\left[[0, T] ; \mathbb{R}^{3}\right]\right) \\
& \quad=\left\{h:[0, T] \longrightarrow \mathbb{R}^{3} \text { continuous and bounded }\right\} \\
& \mathscr{C}\left(\left[[0, T] ; \mathbb{R}^{3}\right]\right) \text { is a Banach space for the norm: }
\end{aligned}
$$

$$
|\|h\||=\sup \{\|h(t)\|, t \in[0, T]\} .
$$

The framework we will refer to for $\Phi$ and $U$ is $\mathbb{R}$, whose norm is denoted by $|\cdot|\left(\right.$ or $\left.\|\cdot\|_{\mathbb{R}}\right)$ :

$$
\mathscr{C}([[0, T] ; \mathbb{R}])
$$

$$
=\{h:[0, T] \longrightarrow \mathbb{R} \text { continuous and bounded }\} .
$$

$\mathscr{C}([[0, T] ; \mathbb{R}])$ is a Banach space for the norm:

$$
|\|h\||=\sup \{|h(t)|, t \in[0, T]\} .
$$

(i) We consider on $\mathbb{R}^{3} \times H_{d}^{m}\left(0, T, \mathbb{R}_{\bar{p}}^{3}\right) \times \mathbb{R} \times \mathbb{R}$ the norm

$$
\|(\overline{\mathrm{E}}, f, \Phi, U)\|=\|\bar{E}\|+\|f\|+|\Phi|+|U| .
$$

(ii) We consider on $\mathscr{C}\left(\left[[0, T] ; \mathbb{R}^{3}\right]\right) \times H_{d}^{3}\left(0, T, \mathbb{R}_{\frac{1}{p}}^{3}\right) \times$ $\mathscr{C}([[0, T] ; \mathbb{R}]) \times \mathscr{C}([[0, T] ; \mathbb{R}])$ the norm:

$$
|\|(\overline{\mathrm{E}}, f, \Phi, U)\||=\|\bar{E}|\|+|\|f\||+|\|\Phi\||+|\|U\|| .
$$

(iii) We will consider the Cauchy problem (59)-(60)-(61)(62) for the initial data:

$$
\begin{array}{ccc}
E^{i}(0)=E_{0}^{i}, & F_{i j}(0)=\varphi_{i j}, \quad f(0, \bar{p})=f_{0}(\bar{p}), \\
\bar{p} \in \mathbb{R}^{3} ; & \Phi(0)=\Phi_{0} ; & U(0)=U_{0},
\end{array}
$$

where $f_{0}$ is given in $H_{d, r}^{3}\left(0, T, \mathbb{R}_{\bar{p}}^{3}\right), E_{0}^{i}, \varphi_{i j}, \Phi_{0} \in \mathbb{R}$, $i, j=1,2,3$, and $U_{0} \in \mathbb{R}^{+}$.

\section{The Local Existence of Solution}

Theorem 9. Let $\tilde{f} \in H_{d, r}^{3}\left(0, T, \mathbb{R}_{\frac{p}{p}}^{3}\right)$ be given, and let $\tilde{\bar{E}}=$ $\left(\widetilde{E^{i}}\right) \in \mathbb{R}^{3}$ be fixed. Then the linearized partial differential equation

$$
\begin{aligned}
\frac{\partial f}{\partial t}+[ & -2 \Gamma_{0 j}^{i} p^{j}+\left(-\widetilde{E^{i}}+\frac{p^{k}}{p^{0}} g^{i j} \varphi_{k j}\right) \\
& \left.\times \int_{\mathbb{R}^{3}} \tilde{f}(t, \bar{p})(\operatorname{det} g)^{1 / 2} d \bar{p}\right] \frac{\partial f}{\partial p^{i}}=\frac{1}{p^{0}} Q(\tilde{f}, \widetilde{f})
\end{aligned}
$$

whose unknown is $f$, with $f(0, \bar{p})=f_{0}$, has in $H_{d}^{3}\left(0, T, \mathbb{R}_{\bar{p}}^{3}\right)$ a local unique and bounded $\star$-weak solution.

Proof. We use the Faedo-Galerkin method in the function space $H_{d}^{3}\left(0, T, \mathbb{R}_{\bar{p}}^{3}\right)$. For the other details, see [1].

Theorem 10. Let $\widetilde{\bar{E}}=\left(\widetilde{E^{i}}\right) \in \mathbb{R}^{3}, i=1,2,3$, be fixed. Then the Boltzmann equation,

$$
\begin{aligned}
\frac{\partial f}{\partial t}+[ & -2 \Gamma_{0 j}^{i} p^{j}+\left(-\widetilde{E^{i}}+\frac{p^{k}}{p^{0}} g^{i j} \varphi_{k j}\right) \\
& \left.\times \int_{\mathbb{R}^{3}} f(t, \bar{p})(\operatorname{det} g)^{1 / 2} d \bar{p}\right] \frac{\partial f}{\partial p^{i}}=\frac{1}{p^{0}} Q(f, f),
\end{aligned}
$$

has in $H_{d}^{3}\left(0, T, \mathbb{R}_{\bar{p}}^{3}\right)$ a local unique $\star$-weak solution $f$ such that $f(0)=f_{0}$. 
Proof. We use the Banach fixed point theorem in $H_{d}^{3}\left(0, T, \mathbb{R}_{\bar{p}}^{3}\right)$ for the map:

$$
\tilde{f} \in H_{d, r}^{3}\left(0, T, \mathbb{R}_{\bar{p}}^{3}\right) \longmapsto \Xi(\tilde{f})=f,
$$

where $f$ satisfies (88).

(i) We firstly prove, using a sequence of approximations $\left(f^{N}\right)$ of $f$, the Banach-Alaoglu theorem and the fact that $H_{d}^{3}\left(0, T, \mathbb{R}_{\bar{p}}^{3}\right)$ is a reflexive space (see [1]) that we can choose $\left\|f_{0}\right\|_{H_{d, r}^{3}\left(0, T, \mathbb{R}_{\bar{p}}^{3}\right)}$ and $T>0$ such that

$$
\tilde{f} \in H_{d, r}^{3}\left(0, T, \mathbb{R}_{\bar{p}}^{3}\right) \Longrightarrow \Xi(\tilde{f})=f \in H_{d, r}^{3}\left(0, T, \mathbb{R}_{\bar{p}}^{3}\right) .
$$

(ii) Let now $\widetilde{f_{1}}, \widetilde{f_{2}} \in H_{d, r}^{3}\left(0, T, \mathbb{R}_{\bar{p}}^{3}\right)$ be given, and let $f_{1}, f_{2}$ be two solutions of (88). Then

$$
\begin{aligned}
& \frac{\partial f_{1}}{\partial t}+\left[-2 \Gamma_{0 j}^{i} p^{j}+\left(-\widetilde{E^{i}}+\frac{p^{k}}{p^{0}} g^{i j} \varphi_{k j}\right)\right. \\
& \left.\quad \times \int_{\mathbb{R}^{3}} \widetilde{f_{1}}(t, \bar{p})(\operatorname{det} g)^{1 / 2} d \bar{p}\right] \frac{\partial f_{1}}{\partial p^{i}}=\frac{1}{p^{0}} Q\left(\widetilde{f_{1}}, \widetilde{f_{1}}\right) \\
& \frac{\partial f_{2}}{\partial t}+\left[-2 \Gamma_{0 j}^{i} p^{j}+\left(-\widetilde{E^{i}}+\frac{p^{k}}{p^{0}} g^{i j} \varphi_{k j}\right)\right. \\
& \left.\quad \times \int_{\mathbb{R}^{3}} \widetilde{f_{2}}(t, \bar{p})(\operatorname{det} g)^{1 / 2} d \bar{p}\right] \frac{\partial f_{2}}{\partial p^{i}}=\frac{1}{p^{0}} Q\left(\widetilde{f_{2}}, \widetilde{f_{2}}\right) .
\end{aligned}
$$

Let $G=f_{1}-f_{2}$ and $\widetilde{G}=\widetilde{f_{1}}-\widetilde{f_{2}}$.

Then we get

$$
\frac{\partial G}{\partial t}+\frac{\widetilde{P}^{i}(\widetilde{G}, \widetilde{\bar{E}})}{p^{0}} \frac{\partial G}{\partial p^{i}}=\frac{1}{p^{0}} Q\left(\widetilde{f_{1}}, \widetilde{G}\right)-\frac{1}{p^{0}} Q\left(\widetilde{G}, \widetilde{f_{2}}\right) .
$$

Conveniently using energy inequalities established in [1], the system (92), and remembering that $G(0, \bar{p})=0$, we obtain

$$
\begin{aligned}
\left\|(1+|\bar{p}|)^{d+|\beta|} \partial_{\bar{p}}^{\beta} G(t)\right\|_{L^{2}} \\
\leq C_{2} \int_{0}^{t} \|(1+|\bar{p}|)^{d+|\beta|} \partial_{\bar{p}}^{\beta} \\
\quad \times\left(\frac{1}{p^{0}} Q\left(\widetilde{f_{1}}, \widetilde{G}\right)-\frac{1}{p^{0}} Q\left(\widetilde{G}, \widetilde{f_{2}}\right)\right) \|_{L^{2}}(\tau) d \tau,
\end{aligned}
$$

where $C_{2}=C\left(g_{i j}^{0}, r, T,\left|E_{0}^{i}\right|,\left|\varphi_{i j}\right|\right)$ is a positive constant.
Then taking the sup in (94), for $t \in[0, T]$ and $|\beta| \leq 3$, we get

$\|G\|_{H_{d}^{3}\left(0, T, \mathbb{R} \frac{3}{p}\right)}$

$$
\begin{aligned}
\leq C \int_{0}^{T} \sup _{t \in[0, T],|\beta| \leq 3}( & \|(1+|\bar{p}|)^{d+|\beta|} \partial_{\bar{p}}^{\beta} \\
\times & \left(\frac{1}{p^{0}} Q\left(\widetilde{f_{1}}, \widetilde{G}\right)\right. \\
& \left.\left.\quad-\frac{1}{p^{0}} Q\left(\widetilde{G}, \widetilde{f_{2}}\right)\right) \|_{L^{2}}\right)(\tau) d \tau
\end{aligned}
$$

which implies

$$
\left.\|G\|_{H_{d}^{3}\left(0, T, \mathbb{R}_{\bar{p}}^{3}\right)} \leq C T\|\widetilde{G}\|_{H_{d}^{3}\left(0, T, \mathbb{R}_{\bar{p}}^{3}\right.}\right)
$$

The relations (91), (96) show clearly that $H_{d}^{3}\left(0, T, \mathbb{R}_{\frac{3}{p}}^{3}\right) \rightarrow$ $H_{d}^{3}\left(0, T, \mathbb{R}_{\bar{p}}^{3}\right), \widetilde{f} \mapsto \Xi(\widetilde{f})=f$ is a contracting map, so by the Banach theorem $\Xi$ has a unique fixed point $f=\tilde{f}$ and the proof of Theorem 10 is complete.

Next, let us introduce the subgroup $G$ of $\mathcal{O}_{3}$ defined by

$$
\begin{aligned}
& G=\left\{N_{\varepsilon, \theta} \in \mathcal{O}_{3},\right. \\
&\left.N_{\varepsilon, \theta}=\left(\begin{array}{ccc}
\varepsilon & 0 & 0 \\
0 & \cos \theta & -\sin \theta \\
0 & \sin \theta & \cos \theta
\end{array}\right), \varepsilon, \theta \in \mathbb{R}, \varepsilon^{2}=1\right\} .
\end{aligned}
$$

A function $g$ on $\mathbb{R}^{3}$ is said to be invariant under $G$ if

$$
g(N \bar{p})=g(\bar{p}) ; \quad \forall N \in G, \forall \bar{p} \in \mathbb{R}^{3} .
$$

Using the observation that $p^{0}$ is invariant under $G$, it is proved in [6] that if $f_{0}$ is invariant under $G$, then so will be the solution $f$ of the Boltzmann equation satisfying $f_{0}(\bar{p})=f(0, \bar{p})$. It is also proved in [7] that $J^{i}=$ $\int_{\mathbb{R}^{3}}\left(p^{i} f(t, \bar{p})(\operatorname{det} g)^{1 / 2} / p^{0}\right) d \bar{p}=0, i=1,2,3$ if and only if $f_{0}$ is invariant under $G$.

One requires in all what follows that the initial datum $f_{0}=$ $f(0 ; \cdot)$ of the distribution function $f$ is not invariant under G. The immediate consequence is that

$$
J^{i}=\int_{\mathbb{R}^{3}} \frac{p^{i} f(t, \bar{p})(\operatorname{det} g)^{1 / 2}}{p^{0}} d \bar{p} \neq 0, \quad i=1,2,3 .
$$

Now, computing the determinant of the system (64), we conclude that, under our requirement, the problem of constraints (64) admits on $[0, T]$ a nontrivial solution:

$$
f \neq 0 ; \quad \varphi_{12}=\varphi_{13}=\varphi_{23}=0,
$$

where $f$ is the unique solution to the Boltzmann equation (60) on $[0, T]$ in which $\tilde{\bar{E}}$ is given. 
Let us now state the following result which shows helpful in what is to follow.

Proposition 11. The Cauchy problem (59)-(60)-(61)-(62) is equivalent to the following problem, for $i=1,2,3$ :

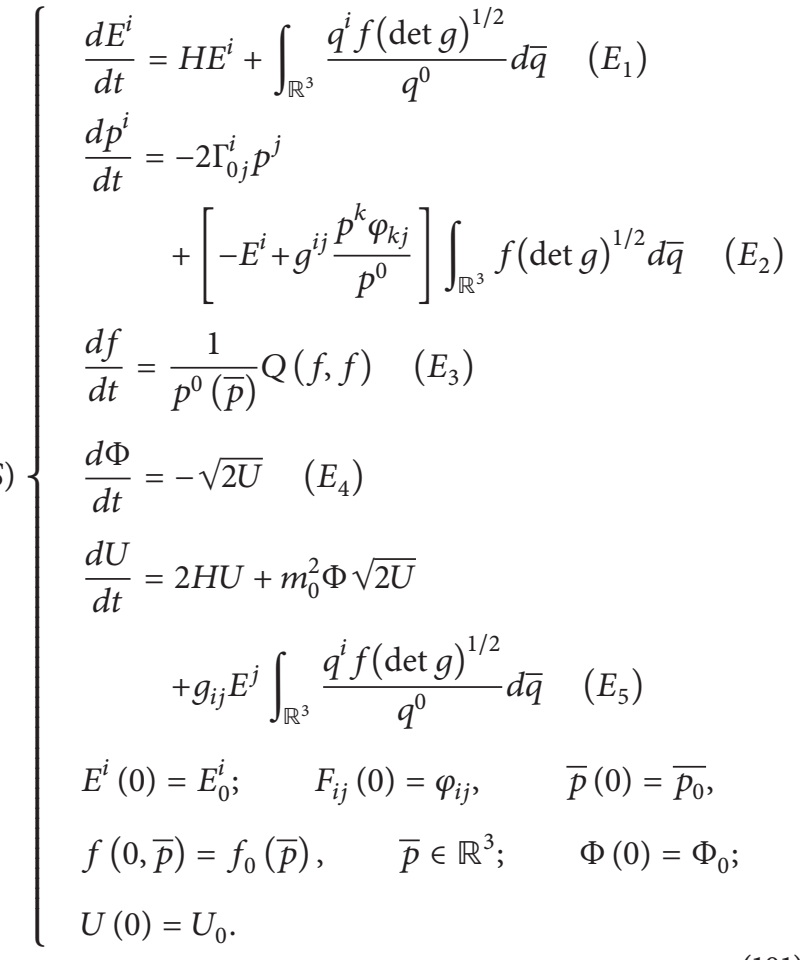

Proof. See [1].

The framework we will refer to for $\bar{p}$ is $\mathbb{R}^{3}$, whose norm is denoted by $\|\cdot\|$ or $\|\cdot\|_{\mathbb{R}^{3}}$.

Let $I=\left(I_{1}, I_{2}, I_{3}, I_{4}, I_{5}\right)$ denote the r.h.s. of $\left(E_{1}\right)-\left(E_{2}\right)$ $\left(E_{3}\right)-\left(E_{4}\right)-\left(E_{5}\right)$, that is,

$$
\begin{gathered}
I_{1}(t, \bar{E}, \bar{p}, f, \Phi, U)=\left(H E^{i}+\int_{\mathbb{R}^{3}} \frac{q^{i} f(\operatorname{det} g)^{1 / 2}}{q^{0}} d \bar{q}\right) \\
I_{2}(t, \bar{E}, \bar{p}, f, \Phi, U) \\
=\left(-2 \Gamma_{0 j}^{i} p^{j}+\left[-E^{i}+g^{i j} \frac{p^{k} \varphi_{k j}}{p^{0}}\right] \int_{\mathbb{R}^{3}} f(\operatorname{det} g)^{1 / 2} d \bar{q}\right) \\
I_{3}(t, \bar{E}, \bar{p}, f, \Phi, U)=\frac{1}{p^{0}(\bar{p})} Q(f, f, \bar{p}) \\
I_{4}(t, \bar{E}, \bar{p}, f, \Phi, U)=\sqrt{2 U} \\
\left.+g_{i j} E^{j} \int_{\mathbb{R}^{3}} \frac{q^{i} f(\operatorname{det} g)^{1 / 2}}{q^{0}} d \bar{q}, f, \Phi, U\right)=2 H U+m_{0}^{2} \Phi \sqrt{2 U} \\
i=1,2,3 .
\end{gathered}
$$

It then appears that, on the contrary to the uncharged case studied in $[6,8]$, the momentum $p=\left(p^{0}, \bar{p}\right)$ also becomes an unknown in the charged case. Note that $f$ and $\bar{p}$ are now independent variables for the system $\left(E_{1}\right)-\left(E_{2}\right)-\left(E_{3}\right)-\left(E_{4}\right)$ $\left(E_{5}\right)$. In this context, the collision operator $Q$ defined by (31) will depend on $\bar{p}$ only through the collision kernel $\sigma$, and we show it by writing now $Q(f, f, \bar{p})$ instead of $Q(f, f)(\bar{p})$. One must from now be careful in order to avoid any confusion between the unknown $\bar{p}$ of the system $\left(E_{1}\right)-\left(E_{2}\right)-\left(E_{3}\right)-\left(E_{4}\right)$ $\left(E_{5}\right)$ and the variable $\bar{p}$ in (26), (27), (57), and (62), for example. For this reason, we denoted the variable in the integrals in $\left(E_{1}\right),\left(E_{2}\right)$, and $\left(E_{5}\right)$ by $\bar{q}$ instead of $\bar{p}$.

Proposition 12. Let $\bar{p}=\left(p^{i}\right), \overline{p_{j}}=\left(p_{j}^{i}\right) \in \mathbb{R}^{3}, j=1,2$, $f \in H_{d}^{3}\left(\mathbb{R}^{3}\right)$ be given. Then

$$
\begin{gathered}
p^{0} \geq \sqrt{g_{i i}}\left|p^{i}\right|, \quad i=1,2,3 \\
\left|\frac{p_{1}^{k}}{p_{1}^{0}}-\frac{p_{2}^{k}}{p_{2}^{0}}\right| \leq B_{1}\left\|\overline{p_{1}}-\overline{p_{2}}\right\| \\
\left|\frac{1}{p_{1}^{0}}-\frac{1}{p_{2}^{0}}\right| \leq B_{2} \frac{\left\|\overline{p_{1}}-\overline{p_{2}}\right\|}{p_{j}^{0}} \\
\left\|\frac{1}{p_{j}^{0}} Q\left(f, f, \overline{p_{1}}\right)-\frac{1}{p_{j}^{0}} Q\left(f, f, \overline{p_{2}}\right)\right\| \leq B_{3}\|f\|\left\|\overline{p_{1}}-\overline{p_{2}}\right\|,
\end{gathered}
$$

where

$$
\begin{gathered}
B_{1}=2 \sum_{i} \sqrt{g_{i i}} \sum_{j} \frac{1}{\sqrt{g_{j j}}}+2 \sum_{i, j} g_{i j}\left(\sum_{k} \frac{1}{\sqrt{g_{k k}}}\right)^{2}+1, \\
B_{2}=2 \sum_{i} \sqrt{g_{i i}}+2 \sum_{i, j} g_{i j} \sum_{k} \frac{1}{\sqrt{g_{k k}}}, \quad B_{3}=B_{3}\left(T ; g_{i j}^{0}\right) .
\end{gathered}
$$

Proof. See [7].

We prove the following.

Proposition 13. Let $\overline{E_{1}}, \overline{E_{2}}, \overline{p_{1}}, \overline{p_{2}} \in \mathbb{R}^{3}, \Phi_{1}, \Phi_{2}, U_{1}, U_{2} \in \mathbb{R}$, $f_{1}, f_{2} \in H_{d}^{3}\left(0, T, \mathbb{R}_{\frac{3}{p}}^{3}\right)$ be given. Then

$$
\begin{gathered}
\left\|I_{1}\left(t, \overline{E_{1}}, \overline{p_{1}}, f_{1}, \Phi_{1}, U_{1}\right)-I_{1}\left(t, \overline{E_{2}}, \overline{p_{2}}, f_{2}, \Phi_{2}, U_{2}\right)\right\|_{\mathbb{R}^{3}} \\
\leq C_{3}\left(\left\|\overline{E_{1}}-\overline{E_{2}}\right\|_{\mathbb{R}^{3}}+\left\|f_{1}-f_{2}\right\|_{H_{d}^{3}\left(0, T, \mathbb{R}_{\bar{p}}^{3}\right)}\right) \\
\left\|I_{2}\left(t, \overline{E_{1}}, \overline{p_{1}}, f_{1}, \Phi_{1}, U_{1}\right)-I_{2}\left(t, \overline{E_{2}}, \overline{p_{2}}, f_{2}, \Phi_{2}, U_{2}\right)\right\|_{\mathbb{R}^{3}} \\
\leq C_{4}\left(\left\|\overline{E_{1}}-\overline{E_{2}}\right\|_{\mathbb{R}^{3}}+\left\|\overline{p_{1}}-\overline{p_{2}}\right\|_{\mathbb{R}^{3}}\right. \\
\left.+\left\|f_{1}-f_{2}\right\|_{H_{d}^{3}\left(0, T, \mathbb{R}_{\bar{p}}^{3}\right)}\right)
\end{gathered}
$$


$\left\|I_{3}\left(t, \overline{E_{1}}, \overline{p_{1}}, f_{1}, \Phi_{1}, U_{1}\right)-I_{3}\left(t, \overline{E_{2}}, \overline{p_{2}}, f_{2}, \Phi_{2}, U_{2}\right)\right\|_{H_{d}^{3}\left(0, T, \mathbb{R}_{\bar{p}}^{3}\right)}$ $\leq C_{5}\left(\left\|\overline{p_{1}}-\overline{p_{2}}\right\|_{\mathbb{R}^{3}}+\left\|f_{1}-f_{2}\right\|_{H_{d}^{3}\left(0, T, \mathbb{R}_{\bar{p}}^{3}\right)}\right)$

$\left\|I_{4}\left(t, \overline{E_{1}}, \overline{p_{1}}, f_{1}, \Phi_{1}, U_{1}\right)-I_{4}\left(t, \overline{E_{2}}, \overline{p_{2}}, f_{2}, \Phi_{2}, U_{2}\right)\right\|_{\mathbb{R}}$ $\leq C_{6}\left\|U_{1}-U_{2}\right\|_{\mathbb{R}}$

$$
\begin{gathered}
\left\|I_{5}\left(t, \overline{E_{1}}, \overline{p_{1}}, f_{1}, \Phi_{1}, U_{1}\right)-I_{5}\left(t, \overline{E_{2}}, \overline{p_{2}}, f_{2}, \Phi_{2}, U_{2}\right)\right\|_{\mathbb{R}} \\
\leq C_{7}\left(\left\|\bar{E}_{1}-\bar{E}_{2}\right\|_{\mathbb{R}^{3}}+\left\|f_{1}-f_{2}\right\|_{H_{d}^{3}\left(0, T, \mathbb{R}_{\bar{p}}^{3}\right)}\right. \\
\left.+\left\|\Phi_{1}-\Phi_{2}\right\|_{\mathbb{R}}+\left\|U_{1}-U_{2}\right\|_{\mathbb{R}}\right),
\end{gathered}
$$

where

$$
\begin{gathered}
C_{3}=C\left(|H|+(\operatorname{det} g)^{1 / 2}\right), \\
C_{4}=C\left(\sum_{i, j} g^{i j}\right)^{2}+C(\operatorname{det} g)^{1 / 2}\left(\left\|f_{1}\right\|+\left\|\overline{E_{2}}\right\|\right) \\
+C(\operatorname{det} g)^{1 / 2} \sum_{i, j} g_{i j} \sum_{k, j}\left|\varphi_{k j}\right|\left(B_{1}+\sum_{k} \frac{1}{\sqrt{g_{k k}}}\right), \\
C_{5}=B_{3}+C\left(T, g_{i j}^{0}\right)(\operatorname{det} g)^{1 / 2}\left(\left\|f_{1}\right\|+\left\|f_{2}\right\|\right)+C B_{2}, \\
C_{6}=\frac{\sqrt{2}}{\sqrt{U_{1}}+\sqrt{U_{2}}}, \\
C_{7}=C|H|+\frac{m_{0}^{2}\left|\Phi_{2}\right| \sqrt{2}}{\sqrt{U_{1}}+\sqrt{U_{2}}}+m_{0}^{2} \sqrt{2 U_{1}} \\
+C(\operatorname{det} g)^{1 / 2} \sum_{i, j} g_{i j}\left(\left\|\bar{E}_{2}\right\|+\left\|f_{1}\right\|\right) .
\end{gathered}
$$

Proof. (a) We have, using (102),

$$
\begin{aligned}
I_{1}\left(t, \overline{E_{1}}, \overline{p_{1}}, f_{1}, \Phi_{1}, U_{1}\right)-I_{1}\left(t, \overline{E_{2}}, \overline{p_{2}}, f_{2}, \Phi_{2}, U_{2}\right) \\
=\left(H\left(E_{1}^{i}-E_{2}^{i}\right)\right. \\
\left.\quad \quad+\int_{\mathbb{R}^{3}} \frac{q^{i}\left(f_{1}(t, \bar{p})-f_{2}(t, \bar{p})\right)(\operatorname{det} g)^{1 / 2}}{q^{0}} d \bar{q}\right) .
\end{aligned}
$$

So by (5) and Proposition 7,

$$
\begin{gathered}
\left|H\left(E_{1}^{i}-E_{2}^{i}\right)+\int_{\mathbb{R}^{3}} \frac{q^{i}\left(f_{1}(t, \bar{p})-f_{2}(t, \bar{p})\right)(\operatorname{det} g)^{1 / 2}}{q^{0}} d \bar{q}\right| \\
\leq|H|\left|E_{1}^{i}-E_{2}^{i}\right|+\frac{C(\operatorname{det} g)^{1 / 2}}{\sqrt{g_{i i}}}\left\|f_{1}-f_{2}\right\|_{L_{d}^{2}\left(\mathbb{R}^{3}\right)^{3}}
\end{gathered}
$$

It follows by (6) that

$$
\begin{aligned}
\left\|I_{1}\left(t, \overline{E_{1}}, \overline{p_{1}}, f_{1}, \Phi_{1}, U_{1}\right)-I_{1}\left(t, \overline{E_{2}}, \overline{p_{2}}, f_{2}, \Phi_{2}, U_{2}\right)\right\|_{\mathbb{R}^{3}} \\
\leq C\left(|H|+(\operatorname{det} g)^{1 / 2}\right) \\
\quad \times\left(\left\|\bar{E}_{1}-\bar{E}_{2}\right\|_{\mathbb{R}^{3}}+\left\|f_{1}-f_{2}\right\|_{H_{d}^{3}\left(\mathbb{R}^{3}\right)}\right) .
\end{aligned}
$$

(b) We still have, by (102),

$$
\begin{aligned}
I_{2}\left(t, \overline{E_{1}}, \overline{p_{1}}, f_{1}, \Phi_{1}, U_{1}\right)-I_{2}\left(t, \overline{E_{2}}, \overline{p_{2}}, f_{2}, \Phi_{2}, U_{2}\right) \\
=\left(2 \Gamma_{0 j}^{i}\left(p_{2}^{j}-p_{1}^{j}\right)\right) \\
+\left((\operatorname{det} g)^{1 / 2} g^{i j} \varphi_{k j}\left[\frac{p_{2}^{k}}{p_{2}^{0}} \int_{\mathbb{R}^{3}} f_{2} d \bar{q}-\frac{p_{1}^{k}}{p_{1}^{0}} \int_{\mathbb{R}^{3}} f_{1} d \bar{q}\right]\right. \\
\left.\quad+(\operatorname{det} g)^{1 / 2}\left[E_{2}^{i} \int_{\mathbb{R}^{3}} f_{2} d \bar{q}-E_{1}^{i} \int_{\mathbb{R}^{3}} f_{1} d \bar{q}\right]\right) .
\end{aligned}
$$

But (5) gives

$$
\left|2 \Gamma_{0 j}^{i}\left(p_{2}^{j}-p_{1}^{j}\right)\right| \leq C\left(\sum_{i, j} g^{i j}\right)^{2}\left\|\overline{p_{1}}-\overline{p_{2}}\right\|_{\mathbb{R}^{3}} .
$$

Invoking Proposition 12, we have

$$
\begin{aligned}
& \left|(\operatorname{det} g)^{1 / 2} g^{i j} \varphi_{k j}\left[\frac{p_{2}^{k}}{p_{2}^{0}} \int_{\mathbb{R}^{3}} f_{2} d \bar{q}-\frac{p_{1}^{k}}{p_{1}^{0}} \int_{\mathbb{R}^{3}} f_{1} d \bar{q}\right]\right| \\
& \quad \leq C(\operatorname{det} g)^{1 / 2}\left(\left\|f_{1}\right\|+\left\|\overline{E_{2}}\right\|\right)\left(\left\|\overline{p_{1}}-\overline{p_{2}}\right\|_{\mathbb{R}^{3}}+\left\|f_{1}-f_{2}\right\|\right),
\end{aligned}
$$$$
\left|(\operatorname{det} g)^{1 / 2}\left[E_{2}^{i} \int_{\mathbb{R}^{3}} f_{2} d \bar{q}-E_{1}^{i} \int_{\mathbb{R}^{3}} f_{1} d \bar{q}\right]\right|
$$

$$
\begin{aligned}
& \leq(\operatorname{det} g)^{1 / 2} \mid g^{i j} \varphi_{k j}\left(\frac{p_{2}^{k}}{p_{2}^{0}}-\frac{p_{1}^{k}}{p_{1}^{0}}\right) \int_{\mathbb{R}^{3}} f_{2} d \bar{q} \\
& +\frac{p_{1}^{k}}{p_{1}^{0}} \int_{\mathbb{R}^{3}}\left(f_{2}-f_{1}\right) d \bar{q} \mid \\
& \leq(\operatorname{det} g)^{1 / 2} \sum_{i, j} g_{i j} \sum_{k, j}\left|\varphi_{k j}\right|
\end{aligned}
$$

$$
\begin{gathered}
\times\left(C B_{1}\left\|f_{1}\right\|\left\|\overline{p_{1}}-\overline{p_{2}}\right\|_{\mathbb{R}^{3}}\right. \\
\left.+\sum_{k} \frac{C}{\sqrt{g_{k k}}}\left\|f_{1}-f_{2}\right\|\right) .
\end{gathered}
$$

So by addition, we conclude that (106) holds. 
(c) We also have, using (102),

$$
\begin{aligned}
I_{3}\left(t, \overline{E_{1}}, \overline{p_{1}}, f_{1}, \Phi_{1}, U_{1}\right)-I_{3}\left(t, \overline{E_{2}}, \overline{p_{2}}, f_{2}, \Phi_{2}, U_{2}\right) \\
=\frac{1}{p_{1}^{0}} Q\left(f_{1}, f_{1}, \overline{p_{1}}\right)-\frac{1}{p_{2}^{0}} Q\left(f_{2}, f_{2}, \overline{p_{2}}\right) \\
=\frac{1}{p_{1}^{0}}\left(Q\left(f_{1}, f_{1}, \overline{p_{1}}\right)-Q\left(f_{2}, f_{2}, \overline{p_{1}}\right)\right) \\
\quad+\frac{1}{p_{1}^{0}}\left(Q\left(f_{2}, f_{2}, \overline{p_{1}}\right)-Q\left(f_{2}, f_{2}, \overline{p_{2}}\right)\right) \\
\quad+\left(\frac{1}{p_{1}^{0}}-\frac{1}{p_{2}^{0}}\right) Q\left(f_{2}, f_{2}, \overline{p_{2}}\right) .
\end{aligned}
$$

By Proposition 6, we have

$$
\begin{gathered}
\left\|\frac{1}{p_{1}^{0}}\left(Q\left(f_{1}, f_{1}, \overline{p_{1}}\right)-Q\left(f_{2}, f_{2}, \overline{p_{1}}\right)\right)\right\|_{H_{d}^{3}\left(0, T, \mathbb{R}_{\bar{p}}^{3}\right)} \\
\leq C\left(T, g_{i j}^{0}\right)\left(\left\|f_{1}\right\|+\left\|f_{2}\right\|\right)\left\|f_{1}-f_{2}\right\| .
\end{gathered}
$$

Using also Proposition 6, we find

$$
\begin{aligned}
& \left\|\frac{1}{p_{1}^{0}}\left(Q\left(f_{2}, f_{2}, \overline{p_{1}}\right)-Q\left(f_{2}, f_{2}, \overline{p_{2}}\right)\right)\right\|_{H_{d}^{3}\left(0, T, \mathbb{R}_{\bar{p}}^{3}\right)} \\
& \leq B_{1}\left\|f_{2}\right\|^{2}\left\|\overline{p_{1}}-\overline{p_{2}}\right\|_{\mathbb{R}^{3}} .
\end{aligned}
$$

Still using Proposition 6 and invoking Proposition 12, we find

$$
\begin{gathered}
\left\|\left(\frac{1}{p_{1}^{0}}-\frac{1}{p_{2}^{0}}\right) Q\left(f_{2}, f_{2}, \overline{p_{2}}\right)\right\|_{H_{d}^{3}\left(0, T, \mathbb{R}_{\bar{p}}^{3}\right)} \\
\leq C B_{2}\left\|f_{2}\right\|^{2}\left\|\overline{p_{1}}-\overline{p_{2}}\right\|_{\mathbb{R}^{3}} .
\end{gathered}
$$

Adding the last three inequalities, we obtain (107).

(d) Similarly, by (102),

$$
\begin{array}{r}
I_{4}\left(t, \overline{E_{1}}, \overline{p_{1}}, f_{1}, \Phi_{1}, U_{1}\right)-I_{4}\left(t, \overline{E_{2}}, \overline{p_{2}}, f_{2}, \Phi_{2}, U_{2}\right) \\
\quad=\sqrt{2 U_{1}}-\sqrt{2 U_{2}}=\frac{\sqrt{2}}{\sqrt{U_{1}}+\sqrt{U_{2}}}\left(U_{1}-U_{2}\right) .
\end{array}
$$

So

$$
\begin{aligned}
& \left|I_{4}\left(t, \overline{E_{1}}, \overline{p_{1}}, f_{1}, \Phi_{1}, U_{1}\right)-I_{4}\left(t, \overline{E_{2}}, \overline{p_{2}}, f_{2}, \Phi_{2}, U_{2}\right)\right| \\
& \quad \leq \frac{\sqrt{2}}{\sqrt{U_{1}}+\sqrt{U_{2}}}\left|U_{1}-U_{2}\right| .
\end{aligned}
$$

(e) Finally by (102) we have

$$
\begin{aligned}
I_{5}\left(t, \overline{E_{1}}, \overline{p_{1}}, f_{1}, \Phi_{1}, U_{1}\right)-I_{5}\left(t, \overline{E_{2}}, \overline{p_{2}}, f_{2}, \Phi_{2}, U_{2}\right) \\
=2 H\left(U_{1}-U_{2}\right)+m_{0}^{2}\left(\Phi_{1} \sqrt{2 U_{1}}-\Phi_{2} \sqrt{2 U_{2}}\right) \\
+\left(g _ { i j } ( \operatorname { d e t } g ) ^ { 1 / 2 } \left(E_{1}^{j} \int_{\mathbb{R}^{3}} \frac{q^{i} f_{1}(t, \bar{p})}{q^{0}} d \bar{q}\right.\right. \\
\left.\left.\quad-E_{2}^{j} \int_{\mathbb{R}^{3}} \frac{q^{i} f_{2}(t, \bar{p})}{q^{0}} d \bar{q}\right)\right) \\
=2 H\left(U_{1}-U_{2}\right)+m_{0}^{2} \Phi_{1}\left(\sqrt{2 U_{1}}-\sqrt{2 U_{2}}\right) \\
+m_{0}^{2} \sqrt{2 U_{2}}\left(\Phi_{1}-\Phi_{2}\right) \\
+g_{i j}(\operatorname{det} g)^{1 / 2} \int_{\mathbb{R}^{3}} \frac{q^{i} f_{1}(t, \bar{p})}{q^{0}} d \bar{q}\left(E_{1}^{j}-E_{2}^{j}\right) \\
+g_{i j} E^{j}\left(\int_{\mathbb{R}^{3}} \frac{q^{i}\left(f_{1}(t, \bar{p})-f_{2}(t, \bar{p})\right)}{q^{0}} d \bar{q}\right) .
\end{aligned}
$$

So by Propositions 6 and 7, using (2) and the inequalities obtained for $I_{1}, I_{2}, I_{4}$,

$$
\begin{gathered}
\left|I_{5}\left(t, \overline{E_{1}}, \overline{p_{1}}, f_{1}, \Phi_{1}, U_{1}\right)-I_{5}\left(t, \overline{E_{2}}, \overline{p_{2}}, f_{2}, \Phi_{2}, U_{2}\right)\right| \\
\leq C_{7}\left(\left\|\bar{E}_{1}-\bar{E}_{2}\right\|_{\mathbb{R}^{3}}+\left\|f_{1}-f_{2}\right\|\right. \\
\left.+\left\|\Phi_{1}-\Phi_{2}\right\|_{\mathbb{R}}+\left\|U_{1}-U_{2}\right\|_{\mathbb{R}}\right),
\end{gathered}
$$

where

$$
\begin{aligned}
C_{7}= & C|H|+\frac{m_{0}^{2}\left|\Phi_{2}\right| \sqrt{2}}{\sqrt{U_{1}}+\sqrt{U_{2}}}+m_{0}^{2} \sqrt{2 U_{1}} \\
& +C(\operatorname{det} g)^{1 / 2} \sum_{i, j} g_{i j}\left(\left\|\bar{E}_{2}\right\|+\left\|f_{1}\right\|\right) .
\end{aligned}
$$

This completes the proof of Proposition 13.

0 ,

One requires in what follows that, for any real number $T>$

$$
U(t)>\frac{U_{0}}{2}, \quad \forall t \in[0, T] .
$$

Theorem 14. Let $t_{0} \geq 0,\left(\overline{E_{t_{0}}}, \overline{p_{t_{0}}}, f_{t_{0}}, \Phi_{t_{0}}, U_{t_{0}}\right) \in \mathbb{R}^{3} \times \mathbb{R}^{3} \times$ $H_{d}^{3}\left(0, T, \mathbb{R}_{\bar{p}}^{3}\right) \times \mathbb{R} \times \mathbb{R}$ be given. Then the following holds.

There exists a real number $\delta>0$ such that the Cauchy problem (S) has a unique solution:

$$
\begin{aligned}
(\bar{E}, \bar{p}, f, \Phi, U) \in & \left(\mathscr{C}\left(\left[\left[t_{0}, t_{0}+\delta\right] ; \mathbb{R}^{3}\right]\right)\right)^{2} \\
& \times H_{d}^{3}\left(t_{0}, t_{0}+\delta, \mathbb{R}_{\bar{p}}^{3}\right) \\
& \times\left(\mathscr{C}\left(\left[\left[t_{0}, t_{0}+\delta\right] ; \mathbb{R}\right]\right)\right)^{2}
\end{aligned}
$$


satisfying $(\bar{E}, \bar{p}, f, \Phi, U)\left(t_{0}\right)=\left(\overline{E_{t_{0}}}, \overline{p_{t_{0}}}, f_{t_{0}}, \Phi_{t_{0}}, U_{t_{0}}\right)$. Moreover, $f$ satisfies the relation:

$$
\|\| f\left\|\mid=\sup \left\{\|f(t)\|, t \in\left[t_{0}, t_{0}+\delta\right]\right\} \leq\right\| f_{t_{0}} \| .
$$

Proof. We apply the standard theory on the first-order differential systems to $\left(E_{1}\right)-\left(E_{2}\right)-\left(E_{3}\right)-\left(E_{4}\right)-\left(E_{5}\right)$.

Since $g_{i j}, \partial_{0} g_{i j}, 1 / g_{i j}, \sigma$ are continuous functions of $t$, so is the function

$$
I(t, \bar{E}, \bar{p}, f, \Phi, U)=\left(I_{1}, I_{2}, I_{3}, I_{4}, I_{5}\right) .
$$

By continuity of the functions $z=g_{i j}, 1 / g_{i j}$ at $t=t_{0}$, there exists a real number $\delta_{0}>0$ such that

$$
t \in] t_{0}-\delta_{0}, t_{0}+\delta_{0}\left[\Longrightarrow|z(t)| \leq\left|z\left(t_{0}\right)\right|+1 .\right.
$$

The previous relation implies, using (5) and (6) to bound $z=g_{i j}, \partial_{0} g_{i j}, 1 / g_{i j}$, that

$$
t \in] t_{0}-\delta_{0}, t_{0}+\delta_{0}\left[\Longrightarrow|z(t)| \leq\left(\sum_{i, j} g_{i j}^{0}+\sum_{i, j} \frac{1}{g_{i j}^{0}}\right) e^{C t_{0}}+1 .\right.
$$

Next, set

$$
\begin{gathered}
B\left(\overline{E_{t_{0}}}, 1\right)=\left\{\bar{E} \in \mathbb{R}^{3},\left\|\bar{E}-\overline{E_{t_{0}}}\right\|<1\right\}, \\
B\left(f_{t_{0}}, 1\right)=\left\{f \in H_{d}^{3}\left(0, T, \mathbb{R}_{\bar{p}}^{3}\right),\left\|f-f_{t_{0}}\right\|<1\right\} .
\end{gathered}
$$

Then

$$
\begin{aligned}
& f \in B\left(f_{t_{0}}, 1\right) \Longrightarrow\|f\| \leq\left\|f_{t_{0}}\right\|+1, \\
& \bar{E} \in B\left(\overline{E_{t_{0}}}, 1\right) \Longrightarrow\|\bar{E}\|<\left\|\overline{E_{t_{0}}}\right\|+1 .
\end{aligned}
$$

Now consider the neighborhood $\left.V_{t_{0}}=\right] t_{0}-\delta_{0}, t_{0}+$ $\delta_{0}\left[\times B\left(\overline{E_{t_{0}}}, 1\right) \times \mathbb{R}^{3} \times B\left(f_{t_{0}}, 1\right) \times\right]-\Phi_{t_{0}}+1, \Phi_{t_{0}}+1[\times]-U_{t_{0}}+$ $1, U_{t_{0}}+1\left[\right.$ of $\left(t_{0}, \overline{E_{t_{0}}}, \overline{p_{t_{0}}}, f_{t_{0}}, \Phi_{t_{0}}, U_{t_{0}}\right)$ in the Banach space $\mathbb{R} \times \mathbb{R}^{3} \times \mathbb{R}^{3} \times H_{d}^{3}\left(0, T, \mathbb{R}_{\bar{p}}^{3}\right) \times \mathbb{R} \times \mathbb{R}$ and take

$$
\left(t, \overline{E_{1}}, \overline{p_{1}}, f_{1}, \Phi_{1}, U_{1}\right),\left(t, \overline{E_{2}}, \overline{p_{2}}, f_{2}, \Phi_{2}, U_{2}\right) \in V_{t_{0}} .
$$

We deduce from the inequalities (105), (106), (107), (108), and (109) the definitions of $C_{3}, C_{4}, C_{5}, C_{6}, C_{7}$, the implications

$$
\begin{gathered}
t \in] t_{0}-\delta_{0}, t_{0}+\delta_{0}[ \\
\Longrightarrow|z(t)| \leq\left(\sum_{i, j} g_{i j}^{0}+\sum_{i, j} \frac{1}{g_{i j}^{0}}\right) e^{C t_{0}}+1, \\
\bar{E} \in B\left(\overline{E_{t_{0}}}, 1\right) \Longrightarrow\|\bar{E}\|<\left\|\overline{E_{t_{0}}}\right\|+1, \\
f \in B\left(f_{t_{0}}, 1\right) \Longrightarrow\|f\| \leq\left\|f_{t_{0}}\right\|+1, \\
\Phi \in]-\Phi_{t_{0}}+1, \Phi_{t_{0}}+1\left[\Longrightarrow|\Phi|<\left|\Phi_{t_{0}}\right|+1,\right. \\
U \in]-U_{t_{0}}+1, U_{t_{0}}+1\left[\Longrightarrow U<U_{t_{0}}+1,\right.
\end{gathered}
$$

and the relation (126) that there exists a constant

$$
C_{8}=C_{8}\left(t_{0}, g_{i j}^{0}, f_{t_{0}},\left|E_{t_{0}}^{i}\right|,\left|\varphi_{i j}\right|,\left|\Phi_{t_{0}}\right|, U_{t_{0}}\right)
$$

such that

$$
\begin{aligned}
& \| I\left(t, \overline{E_{1}}, \overline{p_{1}}, f_{1}, \Phi_{1}, U_{1}\right), \\
& \quad-I\left(t, \overline{E_{2}}, \overline{p_{2}}, f_{2}, \Phi_{2}, U_{2}\right) \|_{\mathbb{R}^{3} \times \mathbb{R}^{3} \times H_{d}^{3}\left(0, T, \mathbb{R}_{\bar{p}}^{3}\right) \times \mathbb{R} \times \mathbb{R}} \\
& \leq C_{8}\left(\left\|\bar{E}_{1}-\bar{E}_{2}\right\|_{\mathbb{R}^{3}}+\left\|\bar{p}_{1}-\bar{p}_{2}\right\|_{\mathbb{R}^{3}}+\left\|f_{1}-f_{2}\right\|_{H_{d}^{3}\left(0, T, \mathbb{R}_{\bar{p}}^{3}\right)}\right. \\
& \left.\quad+\left\|\Phi_{1}-\Phi_{2}\right\|_{\mathbb{R}}+\left\|U_{1}-U_{2}\right\|_{\mathbb{R}}\right)
\end{aligned}
$$

which shows that $I=\left(I_{1}, I_{2}, I_{3}, I_{4}, I_{5}\right)$ is locally Lipschitzian in $(\bar{E}, \bar{p}, f, \Phi, U)$ with respect to the norm of the Banach space $\mathbb{R}^{3} \times \mathbb{R}^{3} \times H_{d}^{3}\left(0, T, \mathbb{R}_{\bar{p}}^{3}\right) \times \mathbb{R} \times \mathbb{R}$. The existence of a unique solution $(\bar{E}, \bar{p}, f, \Phi, U)$ of the differential system $\left(E_{1}\right)-\left(E_{2}\right)$ $\left(E_{3}\right)-\left(E_{4}\right)-\left(E_{5}\right)$ on an interval $\left[t_{0}, t_{0}+\delta\right], \delta>0$, such that $(\bar{E}, \bar{p}, f, \Phi, U)\left(t_{0}\right)=\left(\overline{E_{t_{0}}}, \overline{p_{t_{0}}}, f_{t_{0}}, \Phi_{t_{0}}, U_{t_{0}}\right)$, is guaranteed by the standard theory on the first-order differential systems.

The relation \|\|$f\||=\sup \{\|f(t)\|, t \in[0, \delta]\} \leq|\| f_{t_{0}} \| \mid$ is established in [8].

As a direct consequence, we can deduce that there exists a real number $\delta>0$ such that the Maxwell-BoltzmannEuler system (20)-(21)-(40)-(52) in all Bianchi types 1 to 8 spacetimes has a unique solution $(F, f, \Phi)$ on $[0, \delta]$ satisfying

$$
\begin{aligned}
& F^{0 i}(0)=E_{0}^{i} ; \quad F_{i j}(0)=\varphi_{i j} ; \quad f(0)=f_{0}, \\
& \Phi(0)=\Phi_{0} .
\end{aligned}
$$

\section{The Global Existence}

6.1. The Method. Let $[0, T[$ be the maximal existence domain of solution, denoted here by $(\widetilde{\bar{E}}, \widetilde{\bar{p}}, \widetilde{f}, \widetilde{\Phi}, \widetilde{U})$ and given by Theorem 14, of the system $\left(E_{1}\right)-\left(E_{2}\right)-\left(E_{3}\right)-\left(E_{4}\right)-\left(E_{5}\right)$, with the initial data $\left(\overline{E_{0}}, \overline{p_{0}}, f_{0}, \Phi_{0}, U_{0}\right) \in \mathbb{R}^{3} \times \mathbb{R}^{3} \times H_{d}^{3}\left(0, T, \mathbb{R}_{\bar{p}}^{3}\right) \times \mathbb{R} \times$ $\mathbb{R}$. We intend to prove that $T=+\infty$.

(a) If we already have $T=+\infty$, then the problem of global existence is solved.

(b) We are going to show that if we suppose $T<+\infty$, then the solution $(\tilde{\bar{E}}, \tilde{\bar{p}}, \tilde{f}, \widetilde{\Phi}, \widetilde{U})$ can be extended beyond $T$, which contradicts the maximality of $T$.

(c) The method is as follows: suppose $0<T<+\infty$ and let $t_{0} \in[0, T[$. We want to show that there exists a strictly positive number $\delta>0$ independent of $t_{0}$ such that the system $\left(E_{1}\right)-\left(E_{2}\right)-\left(E_{3}\right)-\left(E_{4}\right)-\left(E_{5}\right)$ on $\left[t_{0}, t_{0}+\delta\right]$, with the initial data $\left(\tilde{\bar{E}}\left(t_{0}\right), \widetilde{\bar{p}}\left(t_{0}\right), \widetilde{f}\left(t_{0}\right), \widetilde{\Phi}\left(t_{0}\right), \widetilde{U}\left(t_{0}\right)\right)$ at $t=t_{0}$, admits a unique solution $(\bar{E}, \bar{p}, f, \Phi, U)$ on $\left[t_{0}, t_{0}+\delta\right]$. Then, by taking $t_{0}$ sufficiently close to $T$, for example, $t_{0}$ such that $0<T-t_{0}<(\delta / 2)$, and hence $T<t_{0}+(\delta / 2)$, we can extend the solution 
$(\tilde{\bar{E}}, \tilde{\bar{p}}, \tilde{f}, \widetilde{\Phi}, \widetilde{U})$ to $\left[0, t_{0}+(\delta / 2)\right]$, which strictly contains $[0, T[$, and this contradicts the maximality of $T$. For the need to simplify the notations, it will be enough if we could look for a number $\delta$ such that $0<\delta<1$.

(d) In what follows we fix a number $r>0$ and we take $f_{0}$ such that $\left\|f_{0}\right\| \leq r$.

By (128) we can write

$$
\left\|\widetilde{f_{t_{0}}}\right\| \leq\left\|f_{0}\right\|
$$

We also have from (139) using (128) that any solution $f$ of the Boltzmann equation on $\left[t_{0}, t_{0}+\delta\right]$ such that $f\left(t_{0}\right)=\widetilde{f}\left(t_{0}\right)$, satisfies the inequality:

$$
\|f(t)\| \leq r, \quad t \in\left[t_{0}, t_{0}+\delta\right] .
$$

Also notice that (140) shows that a solution $(\bar{E}, \bar{p}, f, \Phi, U)$ of the system $\left(E_{1}\right)-\left(E_{2}\right)-\left(E_{3}\right)-\left(E_{4}\right)-\left(E_{5}\right)$ on $\left[t_{0}, t_{0}+\delta\right], \delta>0$, such that $(\bar{E}, \bar{p}, f, \Phi, U)\left(t_{0}\right)=\left(\widetilde{\bar{E}}\left(t_{0}\right)\right.$, $\left.\widetilde{\bar{p}}\left(t_{0}\right), \widetilde{f}\left(t_{0}\right), \widetilde{\Phi}\left(t_{0}\right), \widetilde{U}\left(t_{0}\right)\right)$, satisfies

$$
\begin{aligned}
(\bar{E}, \bar{p}, f, \Phi, U) \in & \left(\mathscr{C}\left(\left[t_{0}, t_{0}+\delta\right] ; \mathbb{R}^{3}\right)\right)^{2} \\
& \times H_{d, r}^{3}\left(t_{0}, t_{0}+\delta, \mathbb{R}^{3}\right) \\
& \times\left(\mathscr{C}\left(\left[t_{0}, t_{0}+\delta\right] ; \mathbb{R}\right)\right)^{2} .
\end{aligned}
$$

In what follows, $[0, T[, T>0$ is the maximal existence domain of solution $(\tilde{\bar{E}}, \widetilde{\bar{p}}, \widetilde{f}, \widetilde{\Phi}, \widetilde{U})$ of $\left(E_{1}\right)-\left(E_{2}\right)-\left(E_{3}\right)-\left(E_{4}\right)-$ $\left(E_{5}\right)$ such that

$$
\begin{aligned}
(\tilde{\bar{E}}, \tilde{\bar{p}}, \widetilde{f}, \widetilde{\Phi}, \widetilde{U})(0) & \\
= & \left(\widetilde{\bar{E}_{0}}, \widetilde{\overline{p_{0}}}, \widetilde{f_{0}}, \widetilde{\Phi_{0}}, \widetilde{U_{0}}\right) \in \mathbb{R}^{3} \times \mathbb{R}^{3} \\
& \times H_{d, r}^{3}\left(0, T, \mathbb{R}_{\bar{p}}^{3}\right) \times \mathbb{R} \times \mathbb{R} .
\end{aligned}
$$

The following result shows helpful in what is to follow.

Lemma 15. $t \mapsto \widetilde{\bar{E}}(t), t \mapsto \widetilde{\bar{p}}(t), t \mapsto \widetilde{U}(t)$, and $t \mapsto \widetilde{\Phi}(t)$ are uniformly bounded over $[0, T[$.

Proof. See [1].

6.2. Global Existence of Solutions. First of all, we consider, for $t_{0} \in[0, T[$ and $\delta>0$,

$$
(\overline{\bar{E}}, \overline{\bar{p}}, \bar{f}) \in\left(\mathscr{C}\left(\left[t_{0}, t_{0}+\delta\right] ; \mathbb{R}^{3}\right)\right)^{2} \times H_{d, r}^{3}\left(t_{0}, t_{0}+\delta, \mathbb{R}^{3}\right) .
$$

Then, we built from system $\left(E_{1}\right)-\left(E_{2}\right)-\left(E_{3}\right)-\left(E_{4}\right)-\left(E_{5}\right)$ by setting in its r.h.s. $I=\left(I_{1}, I_{2}, I_{3}, I_{4}, I_{5}\right)$ which is given by (102) $f=\bar{f}$ in $I_{1}, \bar{E}=\overline{\bar{E}}, f=\bar{f}$ in $I_{2}, \bar{p}=\overline{\bar{p}}$ in $I_{3}$, and $f=\bar{f}$, $\bar{E}=\overline{\bar{E}}$, in $I_{5}$, the following useful differential system:

$$
\begin{aligned}
\frac{d E^{i}}{d t} & =\overline{I_{1}}(t, \bar{E}, \bar{p}, \bar{f}, \Phi, U), \\
\frac{d p^{i}}{d t} & =\overline{I_{2}}(t, \overline{\bar{E}}, \bar{p}, \bar{f}, \Phi, U), \\
\frac{d f}{d t} & =\overline{I_{3}}(t, \bar{E}, \overline{\bar{p}}, f, \Phi, U), \\
\frac{d \Phi}{d t} & =\overline{I_{4}}(t, \bar{E}, \bar{p}, f, \Phi, U), \\
\frac{d U}{d t} & =\overline{I_{5}}(t, \overline{\bar{E}}, \bar{p}, \bar{f}, \Phi, U),
\end{aligned}
$$

where

$$
\begin{gathered}
\overline{I_{1}}(t, \bar{E}, \bar{p}, \bar{f}, \Phi, U)=\left(H E^{i}+\int_{\mathbb{R}^{3}} \frac{q^{i} \bar{f}(\operatorname{det} g)^{1 / 2}}{q^{0}} d \bar{q}\right) \\
\overline{I_{2}}(t, \overline{\bar{E}}, \bar{p}, \bar{f}, \Phi, U) \\
=\left(-2 \Gamma_{0 j}^{i} p^{j}+\left[-\overline{E^{i}}+g^{i j} \frac{p^{k} \varphi_{k j}}{p^{0}}\right] \int_{\mathbb{R}^{3}} \bar{f}(\operatorname{det} g)^{1 / 2} d \bar{q}\right) \\
\overline{I_{3}}(t, \bar{E}, \overline{\bar{p}}, f, \Phi, U)=\frac{1}{p^{0}(\overline{\bar{p}})} Q(f, f, \overline{\bar{p}}) \\
\overline{I_{5}}(t, \overline{\bar{E}}, \bar{p}, \bar{f}, \Phi, U)=2 H U+m_{0}^{2} \Phi \sqrt{2 U} \\
+g_{i j} \overline{E^{j}} \int_{\mathbb{R}^{3}} \frac{q^{i} \bar{f}(\operatorname{det} g)^{1 / 2}}{q^{0}} d \bar{q}, \\
i=1,2,3 .
\end{gathered}
$$

We prove the following.

Proposition 16. Let $t_{0} \in[0, T[, \delta \in] 0,1[$, and $(\overline{\bar{E}}, \overline{\bar{p}}, \bar{f}) \epsilon$ $\left(\mathscr{C}\left(\left[t_{0}, t_{0}+\delta\right] ; \mathbb{R}^{3}\right)\right)^{2} \times H_{d, r}^{3}\left(t_{0}, t_{0}+\delta, \mathbb{R}^{3}\right)$ be given. Then, the differential system (144)-(145)-(146)-(147)-(148) has a unique solution $(\bar{E}, \bar{p}, f, \Phi, U) \in\left(\mathscr{C}\left(\left[t_{0}, t_{0}+\delta\right] ; \mathbb{R}^{3}\right)\right)^{2} \times H_{d, r}^{3}\left(t_{0}, t_{0}+\right.$ $\left.\delta, \mathbb{R}^{3}\right) \times\left(\mathscr{C}\left(\left[t_{0}, t_{0}+\delta\right] ; \mathbb{R}\right)\right)^{2}$ such that $(\bar{E}, \bar{p}, f, \Phi, U)\left(t_{0}\right)=$ $\left(\widetilde{\overline{E_{t_{0}}}}, \widetilde{{\overline{t_{0}}}_{0}}, \widetilde{f_{t_{0}}}, \widetilde{\Phi_{t_{0}}}, \widetilde{U_{t_{0}}}\right)$.

Proof. (a) We consider (144) in $\bar{E}$, with $\overline{I_{1}}$ defined by (149) in which $\bar{f}$ is fixed. Since $g_{i j}, 1 / g_{i j}, \partial_{0} g_{i j}, \bar{f}$ are continuous functions of $t$, so is $\overline{I_{1}}$. Next, we deduce from (105) in which we set $f_{1}=f_{2}=\bar{f}$ that

$$
\begin{aligned}
& \left\|I_{1}\left(t, \overline{E_{1}}, \overline{p_{1}}, \bar{f}, \Phi_{1}, U_{1}\right)-I_{1}\left(t, \overline{E_{2}}, \overline{p_{2}}, \bar{f}, \Phi_{2}, U_{2}\right)\right\|_{\mathbb{R}^{3}} \\
& \quad \leq C_{3}\left\|\bar{E}_{1}-\bar{E}_{2}\right\|,
\end{aligned}
$$

where $C_{3}=C\left(|H|+(\operatorname{det} g)^{1 / 2}\right)$. 
Now we can use (5) and (6) to bound $z=g_{i j}, 1 / g_{i j}, \partial_{0} g_{i j}$ and we obtain, for $t \in\left[t_{0}, t_{0}+\delta\right]$, then $t \leq t_{0}+\delta \leq T+1$,

$$
\begin{array}{r}
|z(t)| \leq\left(C+\sum_{i, j} g_{i j}^{0}\right) e^{C(T+1)}+1, \\
t \in\left[t_{0}, t_{0}+\delta\right], \quad z=g_{i j}, \partial_{0} g_{i j} .
\end{array}
$$

We then deduce from (151) that

$$
C_{3} \leq C_{3}^{\prime}, \quad \text { where } C_{3}^{\prime}=C_{3}^{\prime}\left(g_{i j}^{0}, T\right) \text {. }
$$

By (150) and (152), $\overline{I_{1}}$ is (globally) Lipschitzian with respect to the $\mathbb{R}^{3}$-norm and the local existence of a solution $\bar{E}$ of (144) such that $\bar{E}\left(t_{0}\right)=\widetilde{\bar{E}}\left(t_{0}\right)$ is guaranteed by the standard theory of first-order differential systems.

Now, since $\bar{E}$ satisfies (144) in which $\overline{I_{1}}$ is given by (149), following the same way as in the proof of Lemma 15, substituting $\bar{E}$ to $\widetilde{\bar{E}}, \bar{f}$ to $\widetilde{f}$, and integrating this time over $\left[t_{0}, t_{0}+t\right], t \in[0, \delta[$, lead to

$$
\begin{array}{r}
\left|E^{i}\left(t_{0}+t\right)\right| \leq\left(\left|\widetilde{E^{i}}\left(t_{0}\right)\right|+C_{9}^{i} T\right)+C \int_{t_{0}}^{t_{0}+t}\left|E^{i}\right|(s) d s \\
t \in[0, \delta[, \quad i=1,2,3,
\end{array}
$$

where $C_{9}^{i}=C_{9}^{i}\left(g_{i j}^{0}, T, r,\left|E_{0}^{i}\right|\right)$. However, by Lemma 15, we have, since $t_{0}\left[0, T\left[:\left|\widetilde{E^{i}}\left(t_{0}\right)\right| \leq\left(\left|E_{0}^{i}\right|+C_{9}^{i} T\right) e^{C T}\right.\right.$. Then, by Gronwall inequality,

$$
\begin{array}{r}
\left|E^{i}\left(t_{0}+t\right)\right| \leq\left(\left(\left|E_{0}^{i}\right|+C_{9}^{i} T\right) e^{C T}+C_{11}^{i} T\right) e^{C(T+1)}, \\
t \in[0, \delta[, \quad i=1,2,3,
\end{array}
$$

where (see [1]) $C_{10}^{i}, C_{11}^{i}$ are two constants appearing in Lemma 15 when we bound $t \mapsto \widetilde{\bar{p}}(t)$, which shows that every solution $\bar{E}$ of (144) is uniformly bounded. By the standard theory of first-order differential systems, the solution $\bar{E}$ is defined all over $\left[t_{0}, t_{0}+\delta\left[\right.\right.$ and $\bar{E} \in \mathscr{C}\left(\left[t_{0}, t_{0}+\delta\left[; \mathbb{R}^{3}\right)\right.\right.$.

(b) We also consider (145) in $\bar{p}$, with $\overline{I_{2}}$ defined by (149) in which $\overline{\bar{E}}, \bar{f}$ are fixed. Since $g_{i j}, 1 / g_{i j}, \partial_{0} g_{i j}, \overline{\bar{E}}, \bar{f}$ are continuous functions of $t$, so is $\overline{I_{2}}$. Next, we deduce from (106) in which we set $\overline{E_{1}}=\overline{E_{2}}=\overline{\bar{E}}, f_{1}=f_{2}=\bar{f}$ that

$$
\begin{aligned}
& \left\|I_{2}\left(t, \overline{\bar{E}}, \overline{p_{1}}, \bar{f}, \Phi_{1}, U_{1}\right)-I_{2}\left(t, \overline{\bar{E}}, \overline{p_{2}}, \bar{f}, \Phi_{2}, U_{2}\right)\right\|_{\mathbb{R}^{3}} \\
& \quad \leq C_{4}\left\|\bar{p}_{1}-\bar{p}_{2}\right\|,
\end{aligned}
$$

where

$$
\begin{aligned}
C_{4}= & C\left(\sum_{i, j} g^{i j}\right)^{2}+C(\operatorname{det} g)^{1 / 2}\left(\left\|f_{1}\right\|+\left\|\overline{E_{2}}\right\|\right) \\
& +C(\operatorname{det} g)^{1 / 2} \sum_{i, j} g_{i j} \sum_{k, j}\left|\varphi_{k j}\right|\left(B_{1}+\sum_{k} \frac{1}{\sqrt{g_{k k}}}\right) .
\end{aligned}
$$

Now we can use (5) and (6) to bound $z=g_{i j}, 1 / g_{i j}$ and we obtain, for $t \in\left[t_{0}, t_{0}+\delta\right]$, then $t \leq t_{0}+\delta \leq T+1$,

$$
\begin{array}{r}
|z(t)| \leq\left(\sum_{i, j} g_{i j}^{0}+\sum_{i, j} \frac{1}{g_{i j}^{0}}\right) e^{C(T+1)}+1, \\
t \in\left[t_{0}, t_{0}+\delta\right], \quad z=g_{i j}, \frac{1}{g_{i j}} .
\end{array}
$$

We then deduce from (157), using

$$
\begin{array}{r}
\left|\overline{E^{i}}\left(t_{0}+t\right)\right| \leq\left(\left(\left|E_{0}^{i}\right|+C_{9}^{i} T\right) e^{C T}+C_{11}^{i} T\right) e^{C(T+1)}, \\
t \in[0, \delta[, \quad i=1,2,3,
\end{array}
$$

since $\overline{\bar{E}}=\overline{E_{1}}=\overline{E_{2}}$ is uniformly bounded, and $\|\bar{f}\| \leq\||\bar{f}|\| \leq$ $r$, since $\bar{f} \in H_{d, r}^{3}\left(t_{0}, t_{0}+\delta, \mathbb{R}^{3}\right)$ that

$C_{4} \leq C_{4}^{\prime}, \quad$ where $C_{4}^{\prime}=C_{4}^{\prime}\left(g_{i j}^{0},\left|E_{0}^{i}\right|,\left|\varphi_{i j}\right|, T, r\right)$.

By (155) and (159), $\overline{I_{2}}$ is (globally) Lipschitzian with respect to the $\mathbb{R}^{3}$-norm and the local existence of a solution $\bar{p}$ of (145) such that $\bar{p}\left(t_{0}\right)=\widetilde{\bar{p}}\left(t_{0}\right)$ is guaranteed by the standard theory of first-order differential systems.

Now, since $\bar{p}$ satisfies (152) in which $\overline{I_{2}}$ is given by (149), following the same way as in the proof of Lemma 15, substituting $\overline{\bar{E}}$ to $\widetilde{\bar{E}}, \bar{p}$ to $\widetilde{\bar{p}}$, and $\bar{f}$ to $\tilde{f}$, and integrating this time over $\left[t_{0}, t_{0}+t\right], t \in[0, \delta[$, lead to

$$
\begin{aligned}
\left|p^{i}\left(t_{0}+t\right)\right| \leq & \left(\left|\widetilde{p^{i}}\left(t_{0}\right)\right|+C_{12}^{i} T\right) \\
& +C \int_{t_{0}}^{t_{0}+t}\left|p^{i}\right|(s) d s, \quad t \in[0, \delta[, \quad i=1,2,3,
\end{aligned}
$$

where $C_{12}^{i}=C_{12}^{i}\left(g_{i j}^{0},\left|E_{0}^{i}\right|,\left|\varphi_{i j}\right|, T, r\right)$. However, by Lemma 15, we have, since $t_{0}\left[0, T\left[:\left|\widetilde{p^{i}}\left(t_{0}\right)\right| \leq\left(\left|p_{0}^{i}\right|+C_{8}^{i} T\right) e^{C T}\right.\right.$. Then, by Gronwall inequality,

$$
\begin{array}{r}
\left|p^{i}\left(t_{0}+t\right)\right| \leq\left(\left(\left|p_{0}^{i}\right|+C_{10}^{i} T\right) e^{C T}+C_{12}^{i} T\right) e^{C(T+1)}, \\
t \in[0, \delta[, \quad i=1,2,3,
\end{array}
$$

which shows that every solution $\bar{p}$ of (152) is uniformly bounded. By the standard theory of first-order differential systems, the solution $\bar{p}$ is defined all over $\left[t_{0}, t_{0}+\delta[\right.$ and $\bar{p} \in \mathscr{C}\left(\left[t_{0}, t_{0}+\delta\left[; \mathbb{R}^{3}\right)\right.\right.$.

(c) Next, we have proved in Theorem 10 that the single equation (146) in $f$ has a unique solution $f \in H_{d, r}^{3}\left(t_{0}, t_{0}+\right.$ $\delta, \mathbb{R}^{3}$ ), substituting $t_{0}$ to $0, t_{0}+\delta$ to $T$, such that $f\left(t_{0}\right)=\widetilde{f}\left(t_{0}\right)$.

(d) For (147) in $\Phi$, we have by (108)

$$
\begin{aligned}
& \left\|\overline{I_{4}}\left(t, \overline{E_{1}}, \overline{p_{1}}, f_{1}, \Phi_{1}, U_{1}\right)-\overline{I_{4}}\left(t, \overline{E_{2}}, \overline{p_{2}}, f_{2}, \Phi_{2}, U_{2}\right)\right\|_{\mathbb{R}} \\
& \leq C_{6}^{\prime}\left\|U_{1}-U_{2}\right\|_{\mathbb{R}},
\end{aligned}
$$

where

$$
C_{6}^{\prime}=\frac{\sqrt{2}}{\sqrt{U_{0}}} .
$$

Equations (162) and (163) show that $\overline{I_{4}}$ is (globally) Lipschitzian with respect to the $\mathbb{R}$-norm and the local existence of 
a solution $\Phi$ of (147) such that $\Phi\left(t_{0}\right)=\widetilde{\Phi}\left(t_{0}\right)$ is guaranteed by the standard theory on first-order differential systems.
Since $\Phi$ satisfies (147) in which $\overline{I_{4}}$ is given by (149), substituting $U$ to $\widetilde{U}$ in Lemma 15 , we find

$$
|\dot{\Phi}(t)| \leq \sqrt{2\left(\left(U_{0}+C\left(\left|\widetilde{\bar{E}}_{0}\right|+\sum_{i=1}^{3} C_{9}^{i} T\right) e^{C T} r T\right)+m_{0}^{2} T \max \left\{\Phi_{0}^{2}, \Phi^{2}(T)\right\}\right) e^{C T}}, \quad t \in[0, \delta] .
$$

Applying the Gronwall inequality this time over $\left[t_{0}, t_{0}+t\right]$, $t \in[0, \delta[$, we find

$$
|\Phi(t)| \leq\left|\Phi_{0}\right|+(1+T) \sqrt{2\left(\left(\left(U_{0}+C\left(\left|\widetilde{E}_{0}\right|+\sum_{i=1}^{3} C_{9}^{i} T\right) e^{C T} r T\right)+m_{0}^{2} T \max \left\{\Phi_{0}^{2}, \Phi^{2}(T)\right\}\right) e^{C T}\right)}
$$

and conclude that every solution $\Phi$ of (147) is uniformly bounded. By the standard theory of first-order differential systems, the solution $\Phi$ is defined all over $\left[t_{0}, t_{0}+\delta[\right.$ and $\Phi \in \mathscr{C}\left(\left[t_{0}, t_{0}+\delta[; \mathbb{R})\right.\right.$.

(e) In the end, for (148) in $U$, with $\overline{I_{5}}$ still defined by (149) in which $\overline{\bar{E}}, \bar{f}$ are fixed. Since $g_{i j}, 1 / g_{i j}, \partial_{0} g_{i j}, \overline{\bar{E}}, \bar{f}$ are continuous functions of $t$, so is $\overline{I_{5}}$. We deduce from (109) in which we set $\bar{E}_{1}=\bar{E}_{2}=\overline{\bar{E}}, f_{1}=f_{2}=\bar{f}$ that

$$
\begin{gathered}
\left\|I_{5}\left(t, \overline{\bar{E}}, \overline{p_{1}}, \bar{f}, \Phi_{1}, U_{1}\right)-I_{5}\left(t, \overline{\bar{E}}, \overline{p_{2}}, \bar{f}, \Phi_{2}, U_{2}\right)\right\|_{\mathbb{R}} \\
\leq C_{7}\left(\left\|\Phi_{1}-\Phi_{2}\right\|_{\mathbb{R}}+\left\|U_{1}-U_{2}\right\|_{\mathbb{R}}\right),
\end{gathered}
$$

where

$$
\begin{aligned}
C_{7}=C_{7}= & C|H|+\frac{m_{0}^{2}\left|\Phi_{2}\right| \sqrt{2}}{\sqrt{U_{1}}+\sqrt{U_{2}}}+m_{0}^{2} \sqrt{2 U_{1}} \\
& +C(\operatorname{det} g)^{1 / 2} \sum_{i, j} g_{i j}\left(\left\|\bar{E}_{2}\right\|+\left\|f_{1}\right\|\right) .
\end{aligned}
$$

Invoking (157) and using $\left|\overline{E^{i}}\left(t_{0}+t\right)\right| \leq\left(\left(\left|E_{0}^{i}\right|+C_{9}^{i} T\right) e^{C T}+\right.$ $\left.C_{11}^{i} T\right) e^{C(T+1)}, t \in\left[0, \delta\left[, i=1,2,3\right.\right.$, since $\overline{\bar{E}}=\overline{E_{1}}=\overline{E_{2}}$ is uniformly bounded, $\|\bar{f}\| \leq\||\bar{f}|\| \leq r$, since $\bar{f} \in H_{d, r}^{3}\left(t_{0}, t_{0}+\right.$ $\left.\delta, \mathbb{R}^{3}\right)$, using

$$
|\Phi(t)| \leq\left|\Phi_{0}\right|+(1+T) \sqrt{2\left(\left(\left(U_{0}+C\left(\left|\widetilde{E}_{0}\right|+\sum_{i=1}^{3} C_{9}^{i} T\right) e^{C T} r T\right)+m_{0}^{2} T \max \left\{\Phi_{0}^{2}, \Phi^{2}(T)\right\}\right) e^{C T}\right)}
$$

and Lemma 15, we find

$$
C_{7} \leq C_{7}^{\prime}, \quad \text { where } C_{7}^{\prime}=C_{7}^{\prime}\left(g_{i j}^{0}, T, m_{0},\left|\overline{E_{0}}\right|,\left|\Phi_{0}\right|, U_{0}, r\right) .
$$

We conclude by (166), (169) that $\overline{I_{5}}$ is (globally) Lipschitzian with respect to the $\mathbb{R}$-norm and the local existence of a solution $U$ of (148) such that $U\left(t_{0}\right)=\widetilde{U}\left(t_{0}\right)$ is then guaranteed by the standard theory on first-order differential systems.

We similarly show that every solution $U$ of (148) is uniformly bounded and by the standard theory of first-order differential systems, $U$ is defined all over $\left[t_{0}, t_{0}+\delta[\right.$ and $U \in$ $\mathscr{C}\left(\left[t_{0}, t_{0}+\delta[; \mathbb{R})\right.\right.$.

This ends the proof of Proposition 16.
We now set

$$
\begin{aligned}
X_{\delta}^{t_{0}}= & \left(\mathscr{C}\left(\left[t_{0}, t_{0}+\delta\right] ; \mathbb{R}^{3}\right)\right)^{2} \times H_{d, r}^{3}\left(t_{0}, t_{0}+\delta, \mathbb{R}^{3}\right) \\
Y_{\delta}^{t_{0}}= & \left(\mathscr{C}\left(\left[t_{0}, t_{0}+\delta\right] ; \mathbb{R}^{3}\right)\right)^{2} \times H_{d, r}^{3}\left(t_{0}, t_{0}+\delta, \mathbb{R}^{3}\right) \\
& \times\left(\mathscr{C}\left(\left[t_{0}, t_{0}+\delta\right] ; \mathbb{R}\right)\right)^{2} .
\end{aligned}
$$

$X_{\delta}^{t_{0}}$ is a complete metric subspace of the Banach space $\left(\mathscr{C}\left(\left[t_{0}, t_{0}+\delta\right] ; \mathbb{R}^{3}\right)\right)^{2} \times H_{d}^{3}\left(t_{0}, t_{0}+\delta, \mathbb{R}^{3}\right)$ and $Y_{\delta}^{t_{0}}$ is a complete metric subspace of the Banach space $\left(\mathscr{C}\left(\left[t_{0}, t_{0}+\delta\right] ; \mathbb{R}^{3}\right)\right)^{2} \times$ $H_{d}^{3}\left(t_{0}, t_{0}+\delta, \mathbb{R}^{3}\right) \times\left(\mathscr{C}\left(\left[t_{0}, t_{0}+\delta\right] ; \mathbb{R}\right)\right)^{2}$.

With Proposition 16 we define the map:

$$
g: X_{\delta}^{t_{0}} \longrightarrow Y_{\delta}^{t_{0}}, \quad(\overline{\bar{E}}, \overline{\bar{p}}, \bar{f}) \longmapsto(\bar{E}, \bar{p}, f, \Phi, U)
$$

We claim the following. 
Proposition 17. Let $t_{0}[0, T[$. There exists a number $\delta \in] 0,1[$, independent of $t_{0}$, such that the system $\left(E_{1}\right)-\left(E_{2}\right)-\left(E_{3}\right)-\left(E_{4}\right)$ $\left(E_{5}\right)$ has a unique solution $(\bar{E}, \bar{p}, f, \Phi, U) \in Y_{\delta}^{t_{0}}$ such that $(\bar{E}, \bar{p}, f, \Phi, U)\left(t_{0}\right)=\left(\tilde{\bar{E}}\left(t_{0}\right), \widetilde{\bar{p}}\left(t_{0}\right), \widetilde{f}\left(t_{0}\right), \widetilde{\Phi}\left(t_{0}\right), \widetilde{U}\left(t_{0}\right)\right)$.

Proof. We will prove that there exists a number $\delta \in] 0,1[$, independent of $t_{0}$, such that the map $g$, defined by (172), induces a contraction of the complete metric space $X_{\delta}^{t_{0}}$ defined by $(170)$, which will then have a fixed point $(\bar{E}, \bar{p}, f)$ solution of the system $\left(E_{1}\right)-\left(E_{2}\right)-\left(E_{3}\right)$.

With the initial data $\left(\widetilde{\bar{E}}\left(t_{0}\right), \widetilde{\bar{p}}\left(t_{0}\right), \widetilde{f}\left(t_{0}\right), \widetilde{\Phi}\left(t_{0}\right), \widetilde{U}\left(t_{0}\right)\right)$ at $t=t_{0}$, the differential system (144)-(145)-(146)-(147)-(148) is equivalent for $i=1,2,3$, and using notations (149) to the integral system

$$
\begin{aligned}
& E^{i}\left(t_{0}+t\right)=\widetilde{\bar{E}}\left(t_{0}\right)+\int_{t_{0}}^{t_{0}+t} \overline{I_{1}}(t, \bar{E}, \bar{p}, \bar{f}, \Phi, U)(\tau) d \tau \\
& p^{i}\left(t_{0}+t\right)=\widetilde{p^{i}}\left(t_{0}\right)+\int_{t_{0}}^{t_{0}+t} \overline{I_{2}}(t, \overline{\bar{E}}, \bar{p}, \bar{f}, \Phi, U)(\tau) d \tau \\
& f\left(t_{0}+t\right)=\widetilde{f}\left(t_{0}\right)+\int_{t_{0}}^{t_{0}+t} \overline{I_{3}}(t, \bar{E}, \overline{\bar{p}}, f, \Phi, U)(\tau) d \tau, \\
& \Phi\left(t_{0}+t\right)=\widetilde{\Phi}\left(t_{0}\right)+\int_{t_{0}}^{t_{0}+t} \overline{I_{4}}(t, \bar{E}, \bar{p}, f, \Phi, U)(\tau) d \tau \\
& U\left(t_{0}+t\right)=\widetilde{U}\left(t_{0}\right)+\int_{t_{0}}^{t_{0}+t} I_{5}\left(t, \overline{\bar{E}}, \overline{p_{1}}, \bar{f}, \Phi_{1}, U_{1}\right)(\tau) d \tau
\end{aligned}
$$

To $\left(\overline{\overline{E_{j}}}, \overline{\overline{p_{j}}}, \overline{f_{j}}\right) \in X_{\delta}^{t_{0}}, j=1,2$, correspond the solutions $\left(\overline{E_{j}}, \overline{p_{j}}, f_{j}, \Phi_{j}, U_{j}\right) \in Y_{\delta}^{t_{0}}, j=1,2$, whose existence is proved in Proposition 16. We now write the integral system (173)(174)-(175)-(176)-(177) for $j=1$ and $j=2$, and taking the differences, we obtain the following, with $i=1,2,3$ :

$$
\begin{gathered}
\left(E_{1}^{i}-E_{2}^{i}\right)\left(t_{0}+t\right) \\
=\int_{t_{0}}^{t_{0}+t}\left[I_{1}\left(t, \overline{E_{1}}, \overline{p_{1}}, \overline{f_{1}}, \Phi_{1}, U_{1}\right)\right. \\
\left.\quad-I_{1}\left(t, \overline{E_{2}}, \overline{p_{2}}, \overline{f_{2}}, \Phi_{2}, U_{2}\right)\right](\tau) d \tau, \\
\left(p_{1}^{i}-p_{2}^{i}\right)\left(t_{0}+t\right) \\
=\int_{t_{0}}^{t_{0}+t}\left[I_{2}\left(t, \overline{\overline{E_{1}}}, \overline{p_{1}}, \overline{f_{1}}, \Phi_{1}, U_{1}\right)\right. \\
\left.\quad-I_{2}\left(t, \overline{\overline{E_{2}}}, \overline{p_{2}}, \overline{f_{2}}, \Phi_{2}, U_{2}\right)\right](\tau) d \tau
\end{gathered}
$$

$$
\begin{gathered}
\left(f_{1}-f_{2}\right)\left(t_{0}+t\right) \\
=\int_{t_{0}}^{t_{0}+t}\left[\overline{I_{3}}\left(t, \overline{\overline{E_{1}}}, \overline{\overline{p_{1}}}, f_{1}, \Phi_{1}, U_{1}\right)\right. \\
\left.-\overline{I_{3}}\left(t, \overline{\overline{E_{2}}}, \overline{\overline{p_{2}}}, f_{2}, \Phi_{2}, U_{2}\right)\right](\tau) d \tau, \\
\left(\Phi_{1}-\Phi_{2}\right)\left(t_{0}+t\right) \\
=\int_{t_{0}}^{t_{0}+t}\left[\overline{I_{4}}\left(t, \overline{E_{1}}, \overline{p_{1}}, f_{1}, \Phi_{1}, U_{1}\right)\right. \\
\left.\quad-\overline{I_{4}}\left(t, \overline{E_{2}}, \overline{p_{2}}, f_{2}, \Phi_{2}, U_{2}\right)\right](\tau) d \tau, \\
\left(U_{1}-U_{2}\right)\left(t_{0}+t\right) \\
=\int_{t_{0}}^{t_{0}+t}\left[I_{5}\left(t, \overline{\overline{E_{1}}}, \overline{p_{1}}, \overline{f_{1}}, \Phi_{1}, U_{1}\right)\right. \\
\left.\quad-I_{5}\left(t, \overline{\overline{E_{2}}}, \overline{p_{2}}, \overline{f_{2}}, \Phi_{2}, U_{2}\right)\right](\tau) d \tau .
\end{gathered}
$$

(a) Now we can deduce, from (106) in which we set $f_{1}=$ $\overline{f_{1}}, f_{2}=\overline{f_{2}}$,

$$
\begin{gathered}
\left\|I_{1}\left(t, \overline{E_{1}}, \overline{p_{1}}, \overline{f_{1}}, \Phi_{1}, U_{1}\right)-I_{1}\left(t, \overline{E_{2}}, \overline{p_{2}}, \overline{f_{2}}, \Phi_{2}, U_{2}\right)\right\|_{\mathbb{R}^{3}} \\
\leq C_{3}^{\prime}\left(\left\|\bar{E}_{1}-\bar{E}_{2}\right\|_{\mathbb{R}^{3}}+\left\|\overline{f_{1}}-\overline{f_{2}}\right\|_{H_{d}^{3}\left(\mathbb{R}^{3}\right)}\right)(\tau),
\end{gathered}
$$

where $C_{3}^{\prime}=C_{3}^{\prime}\left(g_{i j}^{0}, T\right)$ is still given by (152).

(b) Next, since $\left(\overline{E_{j}}, \overline{p_{j}}, f_{j}\right) \in X_{\delta}^{t_{0}}$, we deduce from (107) in which we set $\overline{E_{1}}=\overline{\overline{E_{1}}}, \overline{E_{2}}=\overline{\overline{E_{2}}}, f_{1}=\overline{f_{1}}, f_{2}=\overline{f_{2}}$

$$
\begin{gathered}
\left\|I_{2}\left(t, \overline{\overline{E_{1}}}, \overline{p_{1}}, \overline{f_{1}}, \Phi_{1}, U_{1}\right)-I_{2}\left(t, \overline{\overline{E_{2}}}, \overline{p_{2}}, \overline{f_{2}}, \Phi_{2}, U_{2}\right)\right\|_{\mathbb{R}^{3}} \\
\leq C_{4}^{\prime}\left(\left\|\overline{\overline{E_{1}}}-\overline{\overline{E_{2}}}\right\|_{\mathbb{R}^{3}}+\left\|\overline{p_{1}}-\overline{p_{2}}\right\|_{\mathbb{R}^{3}}\right. \\
\left.+\left\|\overline{f_{1}}-\overline{f_{2}}\right\|_{H_{d}^{3}\left(\mathbb{R}^{3}\right)}\right)(\tau),
\end{gathered}
$$

where $C_{4}^{\prime}=C_{4}^{\prime}\left(g_{i j}^{0},\left|E_{0}^{i}\right|,\left|\varphi_{i j}\right|, T, r\right)$ is given by (159).

(c) We have by (108) in which we set $\overline{p_{1}}=\overline{\overline{p_{1}}}, \overline{p_{2}}=$ $\overline{\overline{p_{2}}}$, since $\left(\overline{E_{j}}, \overline{p_{j}}, f_{j}\right) \in X_{\delta}^{t_{0}}$, and using $C_{5}=B_{3}+$ $C\left(T, g_{i j}^{0}\right)(\operatorname{det} g)^{1 / 2}\left(\left\|f_{1}\right\|+\left\|f_{2}\right\|\right)+C B_{2}$ is still given by (108):

$$
\begin{gathered}
\left\|\overline{I_{3}}\left(t, \overline{\overline{E_{1}}}, \overline{\overline{p_{1}}}, f_{1}, \Phi_{1}, U_{1}\right)-\overline{I_{3}}\left(t, \overline{\overline{E_{2}}}, \overline{\overline{p_{2}}}, f_{2}, \Phi_{2}, U_{2}\right)\right\|_{H_{d}^{3}\left(\mathbb{R}^{3}\right)} \\
\leq C_{5}^{\prime}\left(\left\|\overline{\overline{p_{1}}}-\overline{\overline{p_{2}}}\right\|_{\mathbb{R}^{3}}+\left\|f_{1}-f_{2}\right\|_{H_{d}^{3}\left(\mathbb{R}^{3}\right)}\right)(\tau),
\end{gathered}
$$

where $C_{5}^{\prime}=C_{5}^{\prime}\left(g_{i j}^{0}, T, r\right)$. 
(d) We also have, using (109),

$$
\begin{aligned}
& \left\|\overline{I_{4}}\left(t, \overline{E_{1}}, \overline{p_{1}}, f_{1}, \Phi_{1}, U_{1}\right)-\overline{I_{4}}\left(t, \overline{E_{2}}, \overline{p_{2}}, f_{2}, \Phi_{2}, U_{2}\right)\right\|_{\mathbb{R}} \\
& \quad \leq C_{6}^{\prime}\left(\left\|U_{1}-U_{2}\right\|_{\mathbb{R}}\right)(\tau),
\end{aligned}
$$

where $C_{6}^{\prime}$ is given by (163).

(e) Finally, from (126) in which we set $\overline{E_{1}}=\overline{\overline{E_{1}}}, \overline{E_{2}}=\overline{\overline{E_{2}}}$, $f_{1}=\overline{f_{1}}, f_{2}=\overline{f_{2}}$, using the fact that $\overline{\overline{E_{j}}}, \Phi_{j}, U_{j}, j=$ 1,2 are uniformly bounded and $\left\|\overline{f_{j}}(t)\right\| \leq\left\|\overline{f_{j}}\right\| \mid \leq r$, $j=1,2$, we deduce that

$$
\begin{gathered}
\left\|I_{5}\left(t, \overline{\overline{E_{1}}}, \overline{p_{1}}, \overline{f_{1}}, \Phi_{1}, U_{1}\right)-I_{5}\left(t, \overline{\overline{E_{2}}}, \overline{p_{2}}, \overline{f_{2}}, \Phi_{2}, U_{2}\right)\right\|_{\mathbb{R}} \\
\leq C_{7}^{\prime}\left(\left\|\overline{\bar{E}}_{1}-\overline{\bar{E}}_{2}\right\|+\left\|\overline{f_{1}}-\overline{f_{2}}\right\|_{H_{d}^{3}\left(\mathbb{R}^{3}\right)}\right. \\
\left.+\left\|\Phi_{1}-\Phi_{2}\right\|_{\mathbb{R}}+\left\|U_{1}-U_{2}\right\|_{\mathbb{R}}\right)(\tau),
\end{gathered}
$$

where $C_{7}^{\prime}=C_{7}^{\prime}\left(g_{i j}^{0}, T, m_{0},\left|\overline{E_{0}}\right|,\left|\Phi_{0}\right|, U_{0}, r\right)$ is given by (169).

Already notice that the constants $C_{3}^{\prime}, C_{4}^{\prime}, C_{5}^{\prime}, C_{6}^{\prime}$, and $C_{7}^{\prime}$ are absolute constants independent of $t_{0}$.

Now using the inequalities (179), (180), (181), (182), and (183), we deduce from (178), using the norm $|\|\cdot\||$ and since $t \in[0, \delta]$

$$
\begin{gathered}
\left\|\overline{E_{1}}-\overline{E_{2}}\right\| \mid \leq C_{3}^{\prime} \delta\left(\left|\left\|\overline{E_{1}}-\overline{E_{2}}\right\|\right|+\left|\left\|\overline{f_{1}}-\overline{f_{2}}\right\|\right|\right) \\
\left|\left\|\overline{p_{1}}-\overline{p_{2}}\right\|\right| \\
\leq C_{4}^{\prime} \delta\left(\left|\left\|\overline{\overline{E_{1}}}-\overline{\overline{E_{2}}}\right\|\right|+\left|\left\|\overline{p_{1}}-\overline{p_{2}}\right\|\right|+\left|\left\|\overline{f_{1}}-\overline{f_{2}}\right\|\right|\right) \\
\left|\left\|f_{1}-f_{2}\right\|\right| \leq C_{5}^{\prime} \delta\left(\left|\left\|\overline{\overline{p_{1}}}-\overline{\overline{p_{2}}}\right\|\right|+\left|\left\|f_{1}-f_{2}\right\|\right|\right) \\
\left|\left\|\Phi_{1}-\Phi_{2}\right\|\right| \leq C_{6}^{\prime} \delta\left(\left|\left\|U_{1}-U_{2}\right\|\right|\right) \\
\left|\left\|U_{1}-U_{2}\right\|\right| \leq C_{7}^{\prime} \delta\left(\left|\left\|\overline{\overline{E_{1}}}-\overline{\overline{E_{2}}}\right\|\right|+\left|\left\|\overline{f_{1}}-\overline{f_{2}}\right\|\right|\right. \\
\left.+\left|\left\|\Phi_{1}-\Phi_{2}\right\|\right|+\left|\left\|U_{1}-U_{2}\right\|\right|\right) .
\end{gathered}
$$

Now add (184) to obtain

$$
\begin{gathered}
\left|\left\|\bar{E}_{1}-\bar{E}_{2}\right\|\right|+\left|\left\|\bar{p}_{1}-\bar{p}_{2}\right\|\right|+\left|\left\|f_{1}-f_{2}\right\|\right| \\
+\left|\left\|\Phi_{1}-\Phi_{2}\right\|\right|+\left|\left\|U_{1}-U_{2}\right\|\right|
\end{gathered}
$$

$$
\begin{aligned}
\leq & \left(C_{3}^{\prime}+C_{4}^{\prime}+C_{5}^{\prime}+C_{6}^{\prime}+C_{7}^{\prime}\right) \delta \\
& \times\left(\left|\left\|\bar{E}_{1}-\bar{E}_{2}\right\|\right|+\left|\left\|\bar{p}_{1}-\bar{p}_{2}\right\|\right|\right) \\
& +\left(C_{3}^{\prime}+C_{4}^{\prime}+C_{5}^{\prime}+C_{6}^{\prime}+C_{7}^{\prime}\right) \delta \\
& \times\left(\left|\left\|f_{1}-f_{2}\right\|\right|+\left|\left\|\Phi_{1}-\Phi_{2}\right\|\right|+\left|\left\|U_{1}-U_{2}\right\|\right|\right) \\
& +\left(C_{3}^{\prime}+C_{5}^{\prime}+C_{7}^{\prime}\right) \delta \\
& \times\left(\left|\left\|\overline{\bar{E}}_{1}-\overline{\bar{E}}_{2}\right\|\right|+\left|\left\|\overline{\overline{p_{1}}}-\overline{\overline{p_{2}}}\right\|\right|+\left|\left\|\overline{f_{1}}-\overline{f_{2}}\right\|\right|\right) .
\end{aligned}
$$

First of all we take $\delta$ such that

$$
\left(C_{3}^{\prime}+C_{4}^{\prime}+C_{5}^{\prime}+C_{6}^{\prime}+C_{7}^{\prime}\right) \delta<\frac{1}{2} .
$$

Then, reporting (186) in (185) and simplifying, we obtain

$$
\begin{aligned}
\mid \| \bar{E}_{1}- & \bar{E}_{2}\||+|\| \bar{p}_{1}-\bar{p}_{2}\||+|\| f_{1}-f_{2} \| \mid \\
& +\left|\left\|\Phi_{1}-\Phi_{2}\right\|\right|+\left|\left\|U_{1}-U_{2}\right\|\right| \\
\leq & 2\left(C_{3}^{\prime}+C_{5}^{\prime}+C_{7}^{\prime}\right) \delta \\
& \times\left(\left|\left\|\overline{\bar{E}}_{1}-\overline{\bar{E}}_{2}\right\|\right|+\left|\left\|\overline{\overline{p_{1}}}-\overline{\overline{p_{2}}}\right\|\right|+\left|\left\|\overline{f_{1}}-\overline{f_{2}}\right\|\right|\right) .
\end{aligned}
$$

Secondly choosing $\delta$ such that

$$
2\left(C_{3}^{\prime}+C_{5}^{\prime}+C_{7}^{\prime}\right) \delta<\frac{1}{2},
$$

(187) becomes

$$
\begin{aligned}
& \left|\left\|\bar{E}_{1}-\bar{E}_{2}\right\|\right|+\left|\left\|\bar{p}_{1}-\bar{p}_{2}\right\|\right|+\left|\left\|f_{1}-f_{2}\right\|\right| \\
& \quad+\left|\left\|\Phi_{1}-\Phi_{2}\right\|\right|+\left|\left\|U_{1}-U_{2}\right\|\right| \\
& \leq \frac{1}{2}\left(\left|\left\|\overline{\bar{E}}_{1}-\overline{\bar{E}}_{2}\right\|\right|+\left|\left\|\overline{\overline{p_{1}}}-\overline{\overline{p_{2}}}\right\|\right|+\left|\left\|\overline{f_{1}}-\overline{f_{2}}\right\|\right|\right)
\end{aligned}
$$

from which we deduce that

$$
\begin{aligned}
\|\| \bar{E}_{1} & -\bar{E}_{2}\||+|\| \bar{p}_{1}-\bar{p}_{2}\||+|\| f_{1}-f_{2} \| \mid \\
& \leq \frac{1}{2}\left(\left|\left\|\overline{\bar{E}}_{1}-\overline{\bar{E}}_{2}\right\|\right|+\left|\left\|\overline{\overline{p_{1}}}-\overline{\overline{p_{2}}}\right\|\right|+\left|\left\|\overline{f_{1}}-\overline{f_{2}}\right\|\right|\right) .
\end{aligned}
$$

Consequently, if we take

$$
0<\delta<\inf \left\{1, \frac{1}{4\left(C_{3}^{\prime}+C_{4}^{\prime}+C_{5}^{\prime}+C_{6}^{\prime}+C_{7}^{\prime}\right)}\right\},
$$

(190) shows that $g: X_{\delta}^{t_{0}} \rightarrow Y_{\delta}^{t_{0}},(\overline{\bar{E}}, \overline{\bar{p}}, \bar{f}) \mapsto(\bar{E}, \bar{p}, f, \Phi, U)$ defined by (172) induces a contraction $(\overline{\bar{E}}, \overline{\bar{p}}, \bar{f}) \mapsto(\bar{E}, \bar{p}, f)$ in the complete metric space $X_{\delta}^{t_{0}}$ which then has a unique fixed point $(\bar{E}, \bar{p}, f)$ and solution of the integral system (173)(174)-(175) and, hence, of the differential system $\left(E_{1}\right)-\left(E_{2}\right)$ $\left(E_{3}\right)$ such that $(\bar{E}, \bar{p}, f)\left(t_{0}\right)=\left(\tilde{\bar{E}}\left(t_{0}\right), \tilde{\bar{p}}\left(t_{0}\right), \tilde{f}\left(t_{0}\right)\right)$. But using 
the fact that $(\bar{E}, \bar{p}, f) \in\left(\mathscr{C}\left(\left[t_{0}, t_{0}+\delta\right] ; \mathbb{R}^{3}\right)\right)^{2} \times H_{d, r}^{3}\left(t_{0}, t_{0}+\right.$ $\left.\delta, \mathbb{R}^{3}\right)$ and that $t \mapsto \bar{E}(t), t \mapsto \bar{p}(t)$ are uniformly bounded, we conclude by Proposition 16 that the subsystem $\left(E_{4}\right)-\left(E_{5}\right)$ in $(\Phi, U)$ is globally Lipschitzian in $(\Phi, U)$. So the unique solution $(\Phi, U)$, such that $(\Phi, U)\left(t_{0}\right)=\left(\widetilde{\Phi}\left(t_{0}\right), \widetilde{U}\left(t_{0}\right)\right)$, is global on $\left[t_{0}, t_{0}+\delta\right]$ since $\Phi$ and $U$ remain uniformly bounded.

Consequently, for $t_{0} \in[0, T[$, there exists a number $\delta \in$ ]0, 1 [, independent of $t_{0}$, such that the system $\left(E_{1}\right)-\left(E_{2}\right)-\left(E_{3}\right)-$ $\left(E_{4}\right)-\left(E_{5}\right)$ has a unique solution $(\bar{E}, \bar{p}, f, \Phi, U) \in Y_{\delta}^{t_{0}}$ such that

$$
(\bar{E}, \bar{p}, f, \Phi, U)\left(t_{0}\right)=\left(\widetilde{\bar{E}}\left(t_{0}\right), \widetilde{\bar{p}}\left(t_{0}\right), \widetilde{f}\left(t_{0}\right), \widetilde{\Phi}\left(t_{0}\right), \widetilde{U}\left(t_{0}\right)\right) .
$$

This completes the proof of Proposition 17.

Based on the method detailed in Section 6.1, we have proved the following result.

Theorem 18. Let $\overline{E_{0}}=\left(E_{0}^{i}\right) \in \mathbb{R}^{3}, \varphi_{i j} \in \mathbb{R}, f_{0} \in H_{d}^{3}\left(0, T, \mathbb{R} \frac{3}{p}\right)$, $\Phi_{0} \in \mathbb{R}, U_{0} \in \mathbb{R}_{+}^{*}$, be given, such that $\left\|f_{0}\right\| \leq r$, where $r>0$ is a given real number. Then,

(1) the differential system (59)-(60)-(61)-(62) has a unique global solution $(\bar{E}, f, \Phi, U)$ defined all over the interval $[0,+\infty[$ and such that

$$
(\bar{E}, f, \Phi, U)(0)=\left(\overline{E_{0}}, f_{0}, \Phi_{0}, U_{0}\right)
$$

(2) the Maxwell-Boltzmann-Euler system (20)-(21))-(40)(41) in all Bianchi types I to VIII spacetimes has a unique global solution $(F, f, \Phi)$ defined all over the interval $\left[0,+\infty\left[\right.\right.$ and satisfying $\bar{E}(0)=\overline{E_{0}}, F_{i j}(0)=\varphi_{i j}$, $f(0)=f_{0}, \Phi(0)=\Phi_{0}$.

\section{Conclusion}

The physical significance of the work we did in the present paper is the study of the global dynamics of a kind of fast moving, massive, and charged particles, in the case where the gravitational forces are neglected in front of the electromagnetic forces. We have coupled the Maxwell-Boltzmann system with the Euler equations which simply express the conservation of the Stress-matter tensor for the unknown $\Phi$ representing a massive scalar field. Notice that this present work follows our paper titled "Global regular solutions to the Maxwell-Boltzmann-Euler system in a Bianchi type 1 spacetime in presence of a massive scalar field," where the unknowns were like now, the electromagnetic field $F$, subject to the Maxwell equations, the distribution function $f$, subject to the Boltzmann equation, and the massive scalar field $\Phi$, subject to the Euler equations. We have generalized in this present work the result obtained for the Bianchi types 1 spacetime to all Bianchi types 1 to 8 spacetimes. We have only excluded the case of Bianchi type 9 spacetime for the main reason we gave in Section 1 . We have also improved the process to establish energy inequalities and the definition of function spaces. In our future investigations we intend to study the same system coupled this time with the Einstein equations with the cosmological constant. The investigation of the global existence of solutions to this Einstein-MaxwellBoltzmann-Euler system with cosmological constant $\Lambda$ is with a great interest, in the sense that some recent observations show that the whole universe is in an accelerated expansion, and it is the presence of the cosmological constant in the Einstein equations which mathematically shapes this phenomenon, which is also very important for the evolution of our universe and consequently for the humanity.

\section{References}

[1] R. D. Ayissi and N. Noutchegueme, "Global regular solutions to the Maxwell-Boltzmann-Euler system in a Bianch type I spacetime in presence of a massive scalar field," International Journal of Research and Reviews in Applied Sciences, vol. 14, no. 2, pp. 276-315, 2013.

[2] R. D. Ayissi and N. Noutchegueme, "The Faedo-Galerkin method for the relativistic Boltzmann equation in a BIANCHI type I space-time," Communications in Mathematics and Applications, vol. 4, no. 2, 2013.

[3] R. D. Ayissi and N. Noutchegueme, "The Galerkin method for the Global existence of solutions to the Maxwell-Boltzmann system," Applied Physics Research, vol. 5, no. 5, 2013.

[4] R. T. Glassey and W. A. Strauss, "Asymptotic stability of the relativistic Maxwellian," Kyoto University. Research Institute for Mathematical Sciences. Publications, vol. 29, no. 2, pp. 301-347, 1993.

[5] J. Ehlers, A Survey of General Relativity, Astrophysics and Cosmology, E.N.I, 1973.

[6] N. Noutchegueme and D. Dongo, "Global existence of solutions for the Einstein-Boltzmann system in a Bianchi type I spacetime for arbitrarily large initial data," Classical and Quantum Gravity, vol. 23, no. 9, pp. 2979-3003, 2006.

[7] N. Noutchegueme and R. D. Ayissi, "Global existence of solutions to the Maxwell-Boltzmann system in a Bianchi type 1 space-time," Advanced Studies in Theoretical Physics, vol. 4, no. 17-20, pp. 855-878, 2010.

[8] N. Noutchegueme, D. Dongo, and E. Takou, "Global existence of solutions for the relativistic Boltzmann equation with arbitrarily large initial data on a Bianchi type I space-time," General Relativity and Gravitation, vol. 37, no. 12, pp. 2047-2062, 2005. 


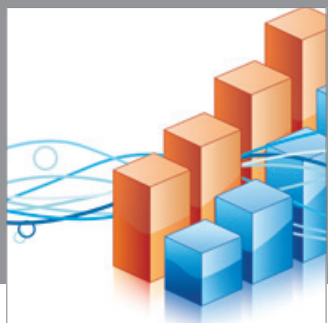

Advances in

Operations Research

mansans

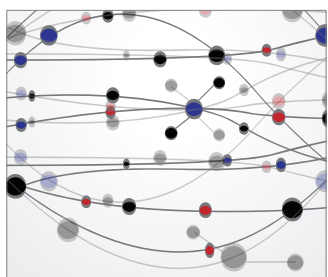

The Scientific World Journal
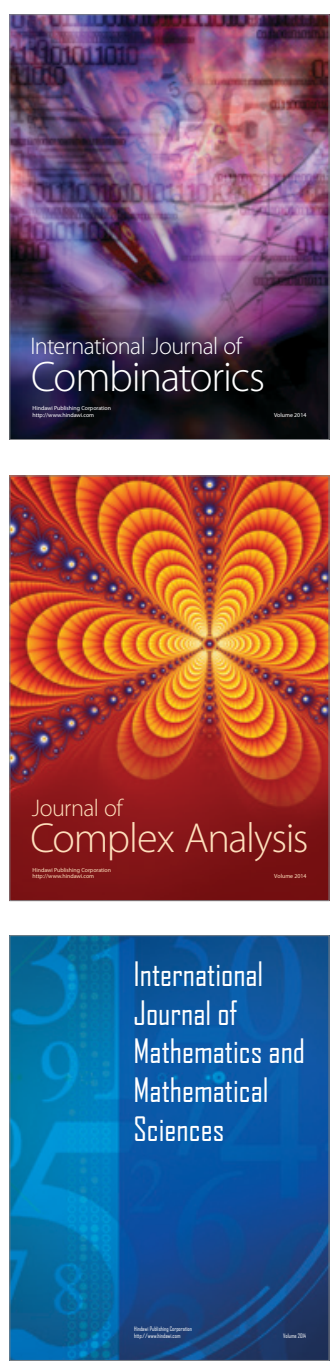
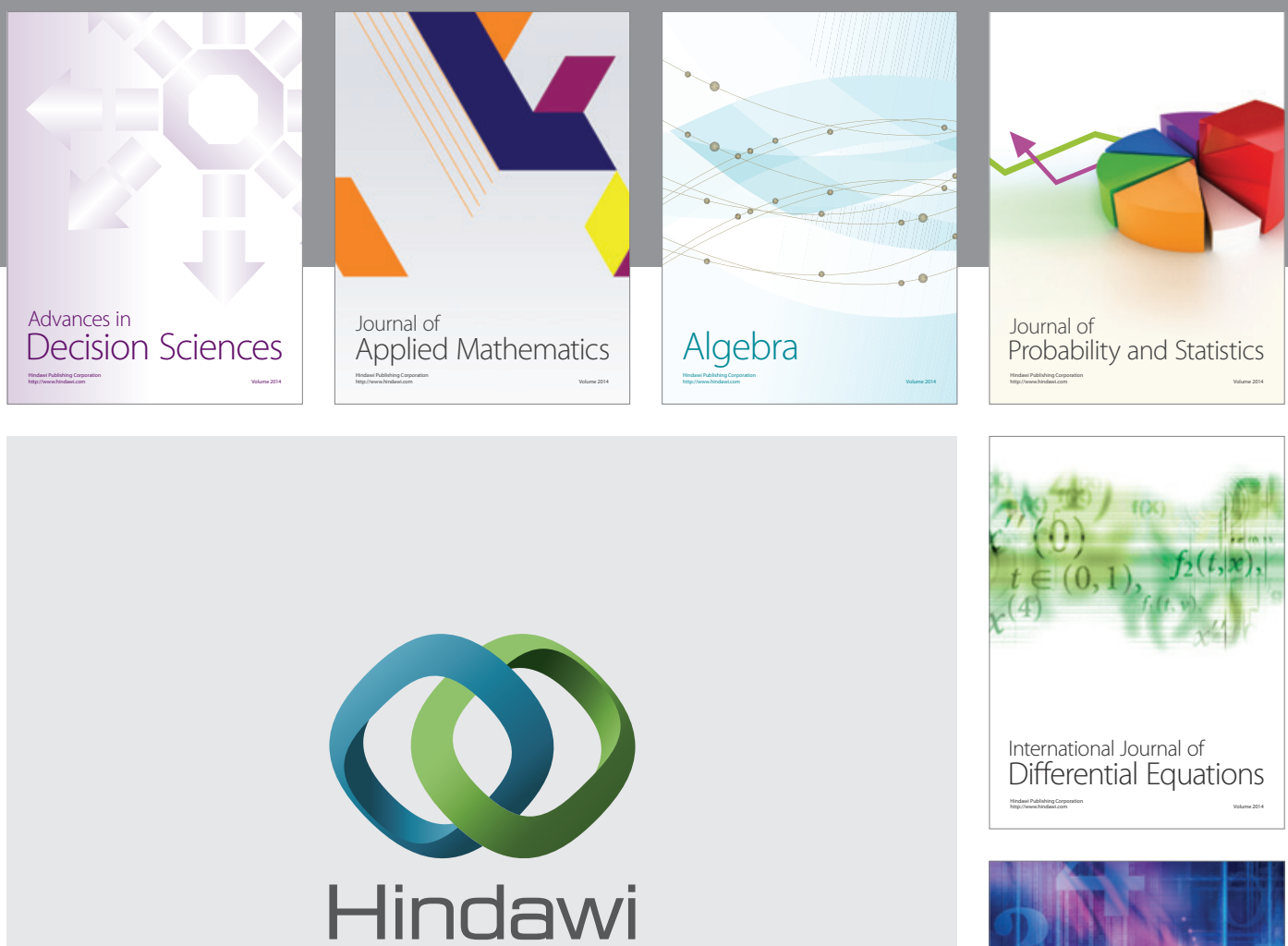

Submit your manuscripts at http://www.hindawi.com
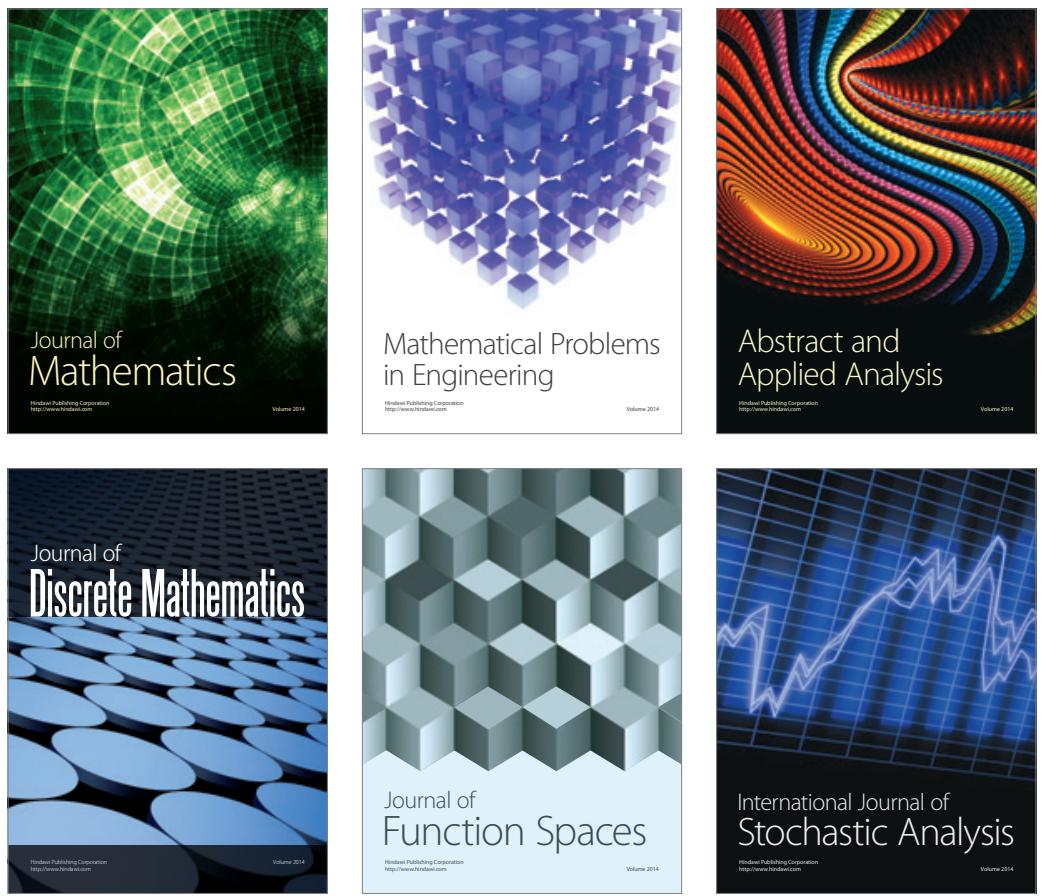

Journal of

Function Spaces

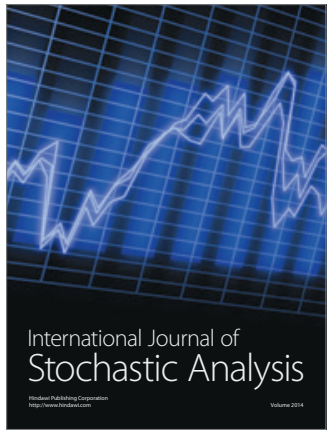

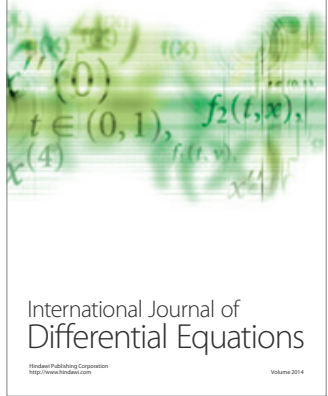
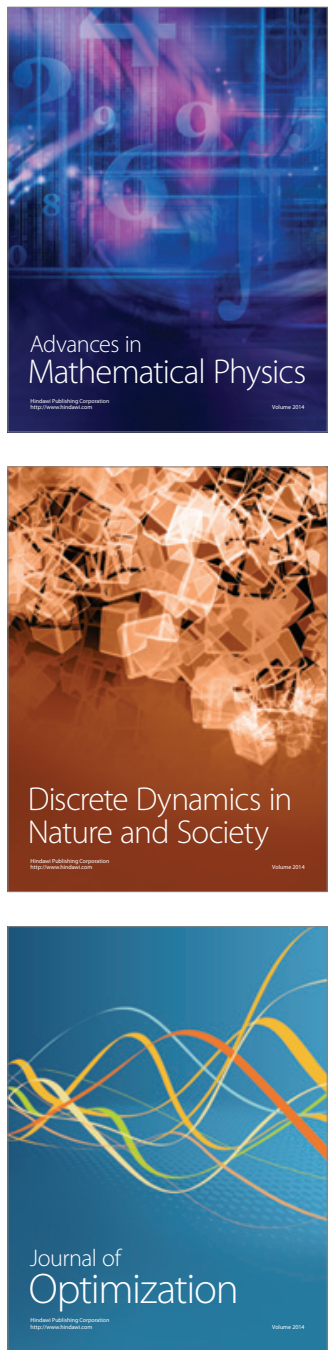